\title{
Unified representation of formulas for single birth processes
}

\author{
Mu-Fa CHEN， Yu-Hui ZHANG \\ School of Mathematical Sciences, Beijing Normal University, Laboratory of Mathematics and \\ Complex Systems, Ministry of Education, Beijing 100875, China
}

(C) Higher Education Press and Springer-Verlag Berlin Heidelberg 2013

\begin{abstract}
Based on a new explicit representation of the solution to the Poisson equation with respect to single birth processes, the unified treatment for various criteria on classical problems (including uniqueness, recurrence, ergodicity, exponential ergodicity, strong ergodicity, as well as extinction probability etc.) for the processes are presented.
\end{abstract}

Keywords Single birth process, Poisson equation, uniqueness, recurrence, ergodicity, moments of return time

MSC $60 \mathrm{~J} 60$

\section{Introduction}

Consider a continuous-time homogeneous Markov chains $\{X(t): t \geqslant 0\}$, on a probability space $(\Omega, \mathscr{F}, \mathbb{P})$, with transition probability matrix $P(t)=\left(p_{i j}(t)\right)$ on a countable state space $\mathbb{Z}_{+}=\{0,1,2, \ldots\}$. We call $\{X(t): t \geqslant 0\}$ a single birth process if its transition rate (density) matrix $Q=\left(q_{i j}: i, j \in \mathbb{Z}_{+}\right)$is irreducible and satisfies that $q_{i, i+1}>0, q_{i, i+j}=0$ for all $i \in \mathbb{Z}_{+}$and $j \geqslant 2$. Such a matrix $Q=\left(q_{i j}\right)$ with $\sum_{j} q_{i j}=0$ for every $i$ (conservativity) is called a single birth $Q$-matrix. Refer to [15]. In the literature, the single birth process is also called upwardly skip-free process, or birth and death process with catastrophes (cf. [1, 2, 3, for instance).

The single birth process, as a natural extension of birth and death process which is a simplest $Q$-process (Markov chain), has its own origins in practice, refer to the earlier papers [2, 13, 15], for instance. The exit boundary of the process consists at most one single extremal point and so the single birth process is nearly the largest class for which the explicit criteria on classical problems can be expected. Actually, the study on the object is quite fruited and relatively

Received March 1, 2014; accepted April 5, 2014

Corresponding author: Yu-Hui ZHANG, E-mail: zhangyh@bnu.edu.cn 
completed (cf. [4, 5, 6, 15, 16, 17]). Based on this advantage, the single birth process becomes a fundamental comparison tool in studying more complex processes, such as infinite-dimensional reaction-diffusion processes. Refer to 4, Chapters 3 and 4, Part III] and [15]. Usually, the single birth process is nonsymmetric and hence it is regarded as a representative one of the non-symmetric processes. For non-symmetric processes, comparing with the symmetric ones, our knowledge is much limited, except for single birth processes to which much results are known as just mentioned. Up to now, the known results are all presented in some recursive forms. This paper introduces a single unified representation, as well as a unified treatment, of various formulas for single birth processes.

Throughout the paper, we consider only the single birth $Q$-matrix $Q=\left(q_{i j}\right)$. Set $q_{i}=-q_{i i}$ for each $i \in \mathbb{Z}_{+}$. For a given function $c$ (to be fixed in this and the next sections, and then to be specified case by case), define an operator $\Omega$ as follows

$$
\Omega g=Q g+c g, \quad \text { where } \quad(Q g)_{i}=\sum_{j} q_{i j}\left(g_{j}-g_{i}\right)
$$

Clearly, if $c \leqslant 0$, then $\Omega$ is an operator corresponding to a single birth process with killing rates $\left(-c_{i}\right)$.

The following sequences are used throughout this paper.

$$
\begin{gathered}
\widetilde{F}_{i}^{(i)}=1, \quad \widetilde{F}_{n}^{(i)}=\frac{1}{q_{n, n+1}} \sum_{k=i}^{n-1} \tilde{q}_{n}^{(k)} \widetilde{F}_{k}^{(i)}, \quad n>i \geqslant 0, \\
\tilde{q}_{n}^{(k)}=q_{n}^{(k)}-c_{n}:=\sum_{j=0}^{k} q_{n j}-c_{n}, \quad 0 \leqslant k<n .
\end{gathered}
$$

Note that if $c \leqslant 0$, then $\tilde{q}_{n}^{(k)} \geqslant 0$ and then $\widetilde{F}_{n}^{(k)} \geqslant 0$ for every $n>k \geqslant 0$. In what follows, we omit the superscript $\sim$ everywhere in $\widetilde{F}$ and $\tilde{q}$ once $c_{i} \equiv 0$, and often use the convention that $\sum_{\varnothing}=0$.

Here is the first of our main results.

Theorem 1.1 Given a single-birth $Q$-matrix $Q=\left(q_{i j}\right)$ and functions $c$ and $f$, the solution $g$ to the Poisson equation

$$
\Omega g=f
$$

has the following representation:

$$
g_{n}=g_{0}+\sum_{0 \leqslant k \leqslant n-1} \sum_{0 \leqslant j \leqslant k} \frac{\widetilde{F}_{k}^{(j)}\left(f_{j}-c_{j} g_{0}\right)}{q_{j, j+1}}, \quad n \geqslant 0 .
$$

In particular, the harmonic function $g$ of $\Omega$ (i.e., $\Omega g=0$ ) can be represented as

$$
g_{n}=g_{0}\left(1-\sum_{0 \leqslant k \leqslant n-1} \sum_{0 \leqslant j \leqslant k} \frac{\widetilde{F}_{k}^{(j)} c_{j}}{q_{j, j+1}}\right), \quad n \geqslant 0 .
$$


Conversely, for each boundary/initial value $g_{0} \in \mathbb{R}$, the function $\left(g_{n}\right)$ defined by (1.4) is a solution to (1.3).

For single birth processes, almost all problems we concerned with are related to the solutions to some specific Poisson equation. Here, we unify these equations as (1.3) with different functions $c$ and $f$ which are listed as follow.

\begin{tabular}{|c|c|c|}
\hline Problem & $c_{i} \in \mathbb{R}$ & $f_{i} \in \mathbb{R}$ \\
\hline Harmonic function & $c_{i} \in \mathbb{R}$ & $f_{i} \equiv 0$ \\
\hline Uniqueness & $c_{i} \equiv-\lambda<0$ & $f_{i} \equiv 0$ \\
\hline Recurrence & $c_{i} \equiv 0$ & $f_{i}=q_{i 0}\left(1-\delta_{i 0}\right)$ \\
\hline Extinction/return probability & $c_{i} \equiv 0$ & $f_{i}=q_{i 0}\left(1-\delta_{i 0}\right)\left(g_{0}-1\right)$ \\
\hline Ergodicity & $c_{i} \equiv 0$ & $f_{i}=q_{i 0}\left(1-\delta_{i 0}\right) g_{0}-1$ \\
\hline Strong ergodicity & $c_{i} \equiv 0$ & $f_{i}=q_{i 0}\left(1-\delta_{i 0}\right) g_{0}-1$ \\
\hline Polynomial moment & $c_{i} \equiv 0$ & $f_{i}^{(\ell)}$ \\
\hline Exponential moment/ergodicity & $c_{i} \equiv \lambda>0$ & $f_{i}=q_{i 0}\left(1-\delta_{i 0}\right)\left(g_{0}-1\right)$ \\
\hline Laplace transform of return time & $c_{i} \equiv-\lambda<0$ & $f_{i}=q_{i 0}\left(1-\delta_{i 0}\right)\left(g_{0}-1\right)$ \\
\hline
\end{tabular}

where $f_{i}^{(\ell)}=q_{i i_{0}}\left(1-\delta_{i i_{0}}\right) g_{i_{0}}-\ell \mathbb{E}_{i} \sigma_{i_{0}}^{\ell-1}$.

We remark that in the two cases for ergodicity and strong ergodicity, even though the Poisson equation and the functions $c$ and $f$ are the same, but their solutions are required to be finite and bounded, respectively.

The paper is organized as follows. The proof of Theorem 1.1 is given in the next section, using a lemma on the representation of solution to a class of linear equations. Then, Sections 3-7 are devoted, respectively, to the criteria on the problems listed in the table above, and related problems to be specific subsequently. Roughly speaking, the unified treatment presented in the paper consists of the following three steps.

(a) Find out the Poisson equation corresponding to the problem we are interested in.

(b) Apply Theorem 1.1 to get the solution to the Poisson equation.

(c) Work out a criterion for the problem using the solution obtained in (b).

Step (a) is more or less known from the previous study; step (b) is now automatic; hence, our main work is spent on step (c).

For the reader's convenience, several key formulas used often in the proofs are collected into an Appendix in a single page which consists the last page of the paper (so that it can be printed out separately). 


\section{The Poisson equation}

In this section, we consider the solutions of the Poisson equation (1.3) for single birth processes. Let us begin with a simple result for the solution to a class of linear equations.

Lemma 2.1 For given real numbers $\left(\alpha_{n k}\right)_{n-1 \geqslant k \geqslant 0}$ and $\left(f_{n}\right)_{n \geqslant 0}$, the solution $\left(g_{n}\right)_{n \geqslant 0}$ to the recursive inhomogeneous equations

$$
g_{n}=\sum_{0 \leqslant k \leqslant n-1} \alpha_{n k} g_{k}+f_{n}, \quad n \geqslant 0
$$

can be represented as

$$
g_{n}=\sum_{0 \leqslant k \leqslant n} \gamma_{n k} f_{k}, \quad n \geqslant 0,
$$

where for fixed $k \geqslant 0,\left(\gamma_{n k}\right)_{n \geqslant k}$ with $\gamma_{k k}=1$ is the solution to the recursive equations

$$
\gamma_{n k}=\sum_{k \leqslant j \leqslant n-1} \alpha_{n j} \gamma_{j k}, \quad n>k .
$$

Proof Use induction. For $n=0$, we have

$$
g_{0}=f_{0}=\gamma_{00} f_{0}=\sum_{0 \leqslant k \leqslant 0} \gamma_{0 k} f_{k} .
$$

Assume that (2.2) holds for all $n \leqslant m$. When $n=m+1$, from (2.1), we see that

$$
\begin{aligned}
g_{m+1} & =\sum_{0 \leqslant k \leqslant m} \alpha_{m+1, k} g_{k}+f_{m+1}=\sum_{0 \leqslant k \leqslant m} \alpha_{m+1, k} \sum_{0 \leqslant \ell \leqslant k} \gamma_{k \ell} f_{\ell}+f_{m+1} \\
& =\sum_{0 \leqslant \ell \leqslant m}\left(\sum_{\ell \leqslant k \leqslant m} \alpha_{m+1, k} \gamma_{k \ell}\right) f_{\ell}+f_{m+1}=\sum_{0 \leqslant \ell \leqslant m} \gamma_{m+1, \ell} f_{\ell}+f_{m+1} \\
& =\sum_{0 \leqslant \ell \leqslant m+1} \gamma_{m+1, \ell} f_{\ell} .
\end{aligned}
$$

Hence, (2.2) holds for $n=m+1$. By induction, the representation (2.2) holds for all $n \geqslant 0$.

Note that the coefficients $\left(\alpha_{n k}\right)$ are often fixed and so are $\left(\gamma_{n k}\right)$. Then Lemma 2.1 says that once replacing $\left(\alpha_{n k}\right)$ by $\left(\gamma_{n k}\right)$, the solution to (2.1) has a complete representation (2.2), mainly in terms of the inhomogeneous term $\left(f_{n}\right)$ in (2.1). 
Without condition $\gamma_{k k}=1$, equation (2.3) is clearly homogeneous. However, it becomes inhomogeneous under condition $\gamma_{k k} \neq 0$ (then one may assume that $\left.\gamma_{k k}=1\right)$ :

$$
\gamma_{n k}=\sum_{k+1 \leqslant j \leqslant n-1} \alpha_{n j} \gamma_{j k}+\alpha_{n k} \gamma_{k k}, \quad n \geqslant k+1
$$

provided $\alpha_{k+1, k} \neq 0$. Otherwise, once $\alpha_{k+1, k}=0$, by induction, we actually have $\gamma_{n k}=0$ for all $n \geqslant k+1$. Thus, under condition $\gamma_{k k}=1$, by Lemma 2.1 (for fixed $k$ ), we have the following alternative representation of $\left(\gamma_{n k}\right)$ :

$$
\gamma_{n k}=\sum_{k+1 \leqslant j \leqslant n} \gamma_{n j} \alpha_{j k}, \quad n \geqslant k+1
$$

In what follows, we will use the following variant of Lemma 2.1, Replacing the initial 0 by $i$ and the coefficient $\left(\alpha_{n k}\right)$ by $\left(\alpha_{n k} \beta_{k}\right)$, respectively, for some non-zero sequence $\left(\beta_{n}\right)$, and set $h_{n}=g_{n} / \beta_{n}(n \geqslant i)$, we obtain the following result.

Corollary 2.2 The solution $\left(h_{n}\right)_{n \geqslant i}$ to the recursive equations

$$
h_{n}=\frac{1}{\beta_{n}}\left(\sum_{i \leqslant k \leqslant n-1} \alpha_{n k} h_{k}+f_{n}\right), \quad n \geqslant i
$$

can be represented as

$$
h_{n}=\sum_{i \leqslant k \leqslant n} \frac{\gamma_{n k}}{\beta_{k}} f_{k}, \quad n \geqslant i
$$

where for each fixed $i,\left(\gamma_{n i}\right)_{n \geqslant i}$ with $\gamma_{i i}=1$ is the solution to the equations

$$
\gamma_{n i}=\frac{1}{\beta_{n}} \sum_{i \leqslant k \leqslant n-1} \alpha_{n k} \gamma_{k i}, \quad n>i .
$$

Equivalently,

$$
\gamma_{i i}=1, \quad \gamma_{n i}=\sum_{i+1 \leqslant k \leqslant n} \frac{\gamma_{n k}}{\beta_{k}} \alpha_{k i}, \quad n \geqslant i+1 .
$$

Specifying $\beta_{n}=q_{n, n+1}$ and $\alpha_{n k}=\tilde{q}_{n}^{(k)}$ in Corollary 2.2 and using the successive formula of $\widetilde{F}_{n}^{(k)}$ defined in (1.1), we obtain the following result.

Corollary 2.3 For given $f$, the sequence $\left(h_{n}\right)$ defined successively by

$$
h_{n}=\frac{1}{q_{n, n+1}}\left(f_{n}+\sum_{i \leqslant k \leqslant n-1} \tilde{q}_{n}^{(k)} h_{k}\right), \quad n \geqslant i
$$


has an unified expression as follows

$$
h_{n}=\sum_{k=i}^{n} \frac{\widetilde{F}_{n}^{(k)}}{q_{k, k+1}} f_{k}, \quad n \geqslant i .
$$

In particular, the sequence $\left(\widetilde{F}_{n}^{(k)}\right)$ defined in (1.1) has the following expression

$$
\widetilde{F}_{i}^{(i)}=1, \quad \widetilde{F}_{n}^{(i)}=\sum_{k=i+1}^{n} \frac{\widetilde{F}_{n}^{(k)} \tilde{q}_{k}^{(i)}}{q_{k, k+1}}, \quad n \geqslant i+1 .
$$

Before moving further, let us mention a comparison result for different $\gamma_{n j}$, which may be useful elsewhere but not in this paper.

Proposition 2.4 For each triple $n \geqslant i>j$, the following assertion holds:

$$
\gamma_{n j}=\sum_{i \leqslant k \leqslant n} \frac{\gamma_{n k}}{\beta_{k}} \sum_{j \leqslant \ell \leqslant i-1} \alpha_{k \ell} \gamma_{\ell j} .
$$

Furthermore, if $\alpha_{n k} \geqslant 0$ and $\beta_{n}>0$ for all $n>k$, then $\gamma_{n i} \gamma_{i j} \leqslant \gamma_{n j}$ for all $n \geqslant i \geqslant j$.

Proof The first assertion is simply a consequence of Corollary 2.2. In fact, for fixed $i>j$, take

$$
f_{n}=\sum_{j \leqslant \ell \leqslant i-1} \alpha_{n \ell} \gamma_{\ell j}, \quad n \geqslant i
$$

Then

$\gamma_{n j}=\frac{1}{\beta_{n}}\left[\sum_{i \leqslant \ell \leqslant n-1} \alpha_{n, \ell} \gamma_{\ell j}+\sum_{j \leqslant \ell \leqslant i-1} \alpha_{n \ell} \gamma_{\ell j}\right]=\frac{1}{\beta_{n}}\left[\sum_{i \leqslant \ell \leqslant n-1} \alpha_{n \ell} \gamma_{\ell j}+f_{n}\right], \quad n \geqslant i$.

Hence, by Corollary 2.2, we get

$$
\gamma_{n j}=\sum_{i \leqslant k \leqslant n} \frac{\gamma_{n k}}{\beta_{k}} f_{k}=\sum_{i \leqslant k \leqslant n} \frac{\gamma_{n k}}{\beta_{k}} \sum_{j \leqslant \ell \leqslant i-1} \alpha_{k \ell} \gamma_{\ell j}, \quad n \geqslant i .
$$

If $\alpha_{n k} \geqslant 0$ and $\beta_{n}>0$ for all $n$ and $k$, then from (2.8), it follows that for all $n>i>j$,

$$
\gamma_{n j}=\gamma_{n i} \gamma_{i j}+\sum_{i+1 \leqslant k \leqslant n} \frac{\gamma_{n k}}{\beta_{k}} \sum_{j \leqslant \ell \leqslant i-1} \alpha_{k \ell} \gamma_{\ell j} \geqslant \gamma_{n i} \gamma_{i j} .
$$

In the cases of $n=i$ or $i=j$, the conclusion is trivial.

Now we turn to prove our first result. 
Proof of Theorem 1.1 For each $i \geqslant 0$, we have

$$
\begin{aligned}
(\Omega g)_{i} & =q_{i, i+1}\left(g_{i+1}-g_{i}\right)-\sum_{0 \leqslant j \leqslant i-1} q_{i j} \sum_{k=j}^{i-1}\left(g_{k+1}-g_{k}\right)+c_{i} g_{i} \\
& =q_{i, i+1}\left(g_{i+1}-g_{i}\right)-\sum_{0 \leqslant k \leqslant i-1} \sum_{j=0}^{k} q_{i j}\left(g_{k+1}-g_{k}\right)+c_{i} g_{i} \\
& =q_{i, i+1}\left(g_{i+1}-g_{i}\right)-\sum_{0 \leqslant k \leqslant i-1}\left(\sum_{j=0}^{k} q_{i j}-c_{i}\right)\left(g_{k+1}-g_{k}\right)+c_{i} g_{0} \\
& =q_{i, i+1}\left(g_{i+1}-g_{i}\right)-\sum_{0 \leqslant k \leqslant i-1} \tilde{q}_{i}^{(k)}\left(g_{k+1}-g_{k}\right)+c_{i} g_{0} .
\end{aligned}
$$

Denote $g_{k+1}-g_{k}$ by $w_{k}$ for $k \geqslant 0$. Then

$$
(\Omega g)_{i}=q_{i, i+1} w_{i}-\sum_{0 \leqslant k \leqslant i-1} \tilde{q}_{i}^{(k)} w_{k}+c_{i} g_{0}, \quad i \geqslant 0 .
$$

Now we rewrite the Poisson equation (1.3) as

$$
w_{i}=\frac{1}{q_{i, i+1}}\left(\sum_{0 \leqslant k \leqslant i-1} \tilde{q}_{i}^{(k)} w_{k}+\tilde{f}_{i}\right), \quad i \geqslant 0
$$

where $\tilde{f}_{i}=f_{i}-c_{i} g_{0}$ for $i \geqslant 0$. By Corollary 2.3, we obtain

$$
w_{i}=\sum_{j=0}^{i} \frac{\widetilde{F}_{i}^{(j)} \tilde{f}_{j}}{q_{j, j+1}}, \quad i \geqslant 0 .
$$

So the solution of the Poisson equation (1.3) satisfies

$$
g_{i}=g_{0}+\sum_{k=0}^{i-1} w_{k}=g_{0}+\sum_{k=0}^{i-1} \sum_{j=0}^{k} \frac{\widetilde{F}_{k}^{(j)} \tilde{f}_{j}}{q_{j, j+1}}, \quad i \geqslant 1 .
$$

The first assertion is proven. The second assertion is simply a consequence of the first one.

To prove the last assertion of the theorem, noting that by (1.4), we have

$$
g_{n+1}-g_{n}=\sum_{j=0}^{n} \frac{\widetilde{F}_{n}^{(j)}\left(f_{j}-c_{j} g_{0}\right)}{q_{j, j+1}}, \quad n \geqslant 0 .
$$

Thus, from (2.9), it follows for each $i \geqslant 0$ that

$$
(\Omega g)_{i}=q_{i, i+1} \sum_{j=0}^{i} \frac{\widetilde{F}_{i}^{(j)}\left(f_{j}-c_{j} g_{0}\right)}{q_{j, j+1}}-\sum_{0 \leqslant k \leqslant i-1} \tilde{q}_{i}^{(k)} \sum_{j=0}^{k} \frac{\widetilde{F}_{k}^{(j)}\left(f_{j}-c_{j} g_{0}\right)}{q_{j, j+1}}+c_{i} g_{0} .
$$


Because (by exchanging the order of sums and using (1.1))

$$
\begin{aligned}
\sum_{0 \leqslant k \leqslant i-1} \tilde{q}_{i}^{(k)} \sum_{j=0}^{k} \frac{\widetilde{F}_{k}^{(j)}\left(f_{j}-c_{j} g_{0}\right)}{q_{j, j+1}} & =\sum_{0 \leqslant j \leqslant i-1} \frac{f_{j}-c_{j} g_{0}}{q_{j, j+1}} \sum_{k=j}^{i-1} \tilde{q}_{i}^{(k)} \widetilde{F}_{k}^{(j)} \\
& =q_{i, i+1} \sum_{0 \leqslant j \leqslant i-1} \frac{\widetilde{F}_{i}^{(j)}\left(f_{j}-c_{j} g_{0}\right)}{q_{j, j+1}}
\end{aligned}
$$

we obtain $\Omega g=f$ as required.

Remark 2.5 (1) One may obtain $\left(\tilde{q}_{n}^{(k)}, \widetilde{F}_{n}^{(k)}\right)$ from $\left(q_{n}^{(k)}, F_{n}^{(k)}\right)$ easily replacing the original $Q=\left(q_{i j}\right)$ by $\widetilde{Q}=\left(\tilde{q}_{i j}\right)$ :

$$
\left\{\begin{array}{l}
\tilde{q}_{i 0}=q_{i 0}-c_{i}, \\
\tilde{q}_{i j}=q_{i j}, \quad j \neq 0, \quad i \in E .
\end{array}\right.
$$

In other words, only the first column of $Q=\left(q_{i j}\right)$ is modified. Then the original Poisson equation $\Omega g=f$ can be rewritten as $\widetilde{Q} g=\tilde{f}$ with $\tilde{f}_{i}=f_{i}-c_{i} g_{0}$.

(2) Alternatively, one may enlarge the space $E$ by adding a point, say -1 for instance. Then introduce suitable $\bar{q}_{-1, i}, \bar{q}_{i,-1}, \bar{g}_{-1}$, and $\bar{f}_{-1}$, so that $\left.\bar{Q}\right|_{E}=Q$, $\left.\bar{g}\right|_{E}=g$, and $\left.\bar{f}\right|_{E}=f$. In this way, one may rewrite $\Omega g=f$ on $E$ as $\bar{Q} \bar{g}=\bar{f}$ on $E \cup\{-1\}$.

(3) To solve the Poisson equation, in view of (2.9), even for the simplest birth-death type, once $c$ appears, it is necessary to go out to the larger class of single birth one, one can not just stay within the class of birth-death processes. Actually, this observation is crucial to solve the Open Problem 9.13 in [7]. Refer to [8, Theorem 2.6].

For the remainder of this section, we consider only the processes on a finite state space $\{0,1, \ldots, N\}$. Note that here the rate $q_{N, N+1}$ is not defined (or setting to be zero), but we allow $c_{N} \neq 0$. Hence $\widetilde{F}_{n}^{(k)}$ is defined up to $n=N-1$ only. The next result is a localized version of Theorem 1.1

Proposition 2.6 Given a single-birth Q-matrix $\left(q_{i j}\right)$ and a function $c$ on the finite state space $\{0,1, \ldots, N\}(N \geqslant 1)$, the following assertions hold.

(i) The solution of the Poisson equation $\Omega g=f$ has the following form:

$$
g_{n}=g_{0}+\sum_{0 \leqslant k \leqslant n-1} \sum_{0 \leqslant j \leqslant k} \frac{\widetilde{F}_{k}^{(j)}\left(f_{j}-c_{j} g_{0}\right)}{q_{j, j+1}}, \quad 0 \leqslant n \leqslant N,
$$

with boundary condition

$$
c_{N} g_{0}=\sum_{k=0}^{N-1} \tilde{q}_{N}^{(k)} \sum_{j=0}^{k} \frac{\widetilde{F}_{k}^{(j)}\left(f_{j}-c_{j} g_{0}\right)}{q_{j, j+1}}+f_{N} .
$$


(ii) Let $c \leqslant 0$. Then the harmonic equation $\Omega g=0$ has only the trivial solution $g_{i} \equiv 0$ iff there exists some $c_{i}<0$.

(iii) The unique solution $g$ to the equation $\left.\Omega g\right|_{\{0,1, \ldots, N-1\}}=0$ (locally harmonic) with $g_{0}=1$ is as follows:

$$
g_{n}=1-\sum_{0 \leqslant k \leqslant n-1} \sum_{0 \leqslant j \leqslant k} \frac{\widetilde{F}_{k}^{(j)} c_{j}}{q_{j, j+1}}, \quad 0 \leqslant n \leqslant N
$$

which is increasing once $c \leqslant 0$.

Proof (a) The proof is nearly the same as the one of Theorem 1.1, except we have to take care for the boundary at $N$. By (2.9), for $0 \leqslant i \leqslant N-1$, we have

$$
(\Omega g)_{i}=q_{i, i+1}\left(g_{i+1}-g_{i}\right)-\sum_{0 \leqslant k \leqslant i-1} \tilde{q}_{i}^{(k)}\left(g_{k+1}-g_{k}\right)+c_{i} g_{0} .
$$

Denote $g_{k+1}-g_{k}$ by $w_{k}$ for all $0 \leqslant k<N$. Then

$$
\begin{aligned}
& (\Omega g)_{i}=q_{i, i+1} w_{i}-\sum_{0 \leqslant k \leqslant i-1} \tilde{q}_{i}^{(k)} w_{k}+c_{i} g_{0}, \quad 0 \leqslant i<N \\
& (\Omega g)_{N}=-\sum_{k=0}^{N-1} \tilde{q}_{N}^{(k)} w_{k}+c_{N} g_{0} .
\end{aligned}
$$

Rewrite the Poisson equation as

$$
w_{i}=\frac{1}{q_{i, i+1}}\left(\tilde{f}_{i}+\sum_{0 \leqslant k \leqslant i-1} \tilde{q}_{i}^{(k)} w_{k}\right), \quad 0 \leqslant i<N,
$$

where $\tilde{f}_{i}=f_{i}-c_{i} g_{0}$ for all $0 \leqslant i \leqslant N$. By Corollary 2.3 , we get

$$
w_{i}=\sum_{j=0}^{i} \frac{\widetilde{F}_{i}^{(j)} \tilde{f}_{j}}{q_{j, j+1}}, \quad 0 \leqslant i<N .
$$

So the solution of the Poisson equation satisfies

$$
g_{i}=g_{0}+\sum_{k=0}^{i-1} w_{k}=g_{0}+\sum_{k=0}^{i-1} \sum_{j=0}^{k} \frac{\widetilde{F}_{k}^{(j)} \tilde{f}_{j}}{q_{j, j+1}}, \quad 1 \leqslant i \leqslant N .
$$

Combining this with the boundary condition $(\Omega g)_{N}=f_{N}$ and (2.13), we obtain the first assertion.

(b) We have just seen that the harmonic solution $g$ satisfies

$$
g_{n}=g_{0}\left(1-\sum_{k=0}^{n-1} \sum_{j=0}^{k} \frac{\widetilde{F}_{k}^{(j)} c_{j}}{q_{j, j+1}}\right), \quad 1 \leqslant n \leqslant N .
$$


and

$$
g_{0}\left(c_{N}+\sum_{k=0}^{N-1} \tilde{q}_{N}^{(k)} \sum_{j=0}^{k} \frac{\widetilde{F}_{k}^{(j)} c_{j}}{q_{j, j+1}}\right)=0
$$

When $c \leqslant 0$, by irreducibility, we have not only $\tilde{q}_{N}^{(N-1)}>0$ but also $\widetilde{F}_{N-1}^{(j)}>0$ for every $j: 0 \leqslant j \leqslant N-1$. Hence, if there exists some $c_{i}<0$, then we must have $g_{0}=0$ by the last equation. Furthermore, by (2.14), we indeed have $g \equiv 0$.

Conversely, if $c_{i} \equiv 0$, then every constant function $g \neq 0$ is a solution to the equation $\Omega g=0$. Hence the harmonic function $g$ can be non-trivial.

(c) To prove the third assertion, based on the second one, we have to use a smaller space $\{0,1, \ldots, N-1\}$ instead of the original $\{0,1, \ldots, N\}$ to avoid the trivial solution. The assertion now follows from (2.14).

The next result is exceptional of the paper. Instead of single birth, we consider single death processes on a finite state space. The result may be regarded as a dual of Proposition 2.6. It indicates that a large parts of the study in the paper is meaningful for the single death processes, but we will not go to the details here.

A matrix $Q=\left(q_{i j}\right)$ is called of single death if $q_{i, i-j}>0$ iff $j=1$ for $i \geqslant 1$.

Proposition 2.7 Given a single death $Q$-matrix $Q=\left(q_{i j}\right)$ and a function $\left(c_{i}\right)$ on the finite state space $\{0,1, \ldots, N\}$, define $\tilde{q}_{n}^{(k)}=\sum_{j=k}^{N} q_{n j}-c_{n}$ for $k>n$ and

$$
\widetilde{F}_{i}^{(i)}=1, \quad \widetilde{F}_{n}^{(i)}=\frac{1}{q_{n, n-1}} \sum_{k=n+1}^{i} \tilde{q}_{n}^{(k)} \widetilde{F}_{k}^{(i)}, \quad 1 \leqslant n<i .
$$

Then

(i) the solution $g$ to the Poisson equation $\Omega g=f$ has the following representation:

$$
g_{n}=g_{N}+\sum_{n+1 \leqslant k \leqslant N} \sum_{k \leqslant j \leqslant N} \frac{\widetilde{F}_{k}^{(j)}\left(f_{j}-c_{j} g_{N}\right)}{q_{j, j-1}}, \quad 0 \leqslant n \leqslant N
$$

with boundary condition

$$
c_{0} g_{N}=\sum_{k=1}^{N} \tilde{q}_{0}^{(k)} \sum_{j=k}^{N} \frac{\widetilde{F}_{k}^{(j)}\left(f_{j}-c_{j} g_{N}\right)}{q_{j, j-1}}+f_{0} .
$$

(ii) The unique solution with $g_{N}=1$ to equation $\left.Q g\right|_{\{1,2, \ldots, N\}}=0$ is as follows:

$$
g_{n}=1-\sum_{n+1 \leqslant k \leqslant N} \sum_{k \leqslant j \leqslant N} \frac{\widetilde{F}_{k}^{(j)} c_{j}}{q_{j, j-1}} \quad(0 \leqslant n \leqslant N)
$$

which is decreasing in $n$ once $c \leqslant 0$. 
Proof For $1 \leqslant i \leqslant N$, we have

$$
\begin{aligned}
(\Omega g)_{i} & =q_{i, i-1}\left(g_{i-1}-g_{i}\right)+\sum_{i+1 \leqslant j \leqslant N} q_{i j} \sum_{k=i+1}^{j}\left(g_{k}-g_{k-1}\right)+c_{i} g_{i} \\
& =q_{i, i-1}\left(g_{i-1}-g_{i}\right)+\sum_{i+1 \leqslant k \leqslant N} \sum_{j=k}^{N} q_{i j}\left(g_{k}-g_{k-1}\right)+c_{i} g_{i} \\
& =q_{i, i-1}\left(g_{i-1}-g_{i}\right)-\sum_{i+1 \leqslant k \leqslant N} \tilde{q}_{i}^{(k)}\left(g_{k-1}-g_{k}\right)+c_{i} g_{N} .
\end{aligned}
$$

Denote $g_{k-1}-g_{k}$ by $w_{k}$ for all $1 \leqslant k \leqslant N$. Then

$$
\begin{gathered}
(\Omega g)_{i}=q_{i, i-1} w_{i}-\sum_{i+1 \leqslant j \leqslant N} \tilde{q}_{i}^{(k)} w_{k}+c_{i} g_{N}, \quad 1 \leqslant i \leqslant N \\
(\Omega g)_{0}=-\sum_{k=1}^{N} \tilde{q}_{0}^{(k)} w_{k}+c_{0} g_{N} .
\end{gathered}
$$

Now we rewrite the Poisson equation as

$$
w_{i}=\frac{1}{q_{i, i-1}}\left(\tilde{f}_{i}+\sum_{i+1 \leqslant j \leqslant N} q_{i}^{(k)} w_{k}\right), \quad 1 \leqslant i \leqslant N
$$

where $\tilde{f}_{i}=f_{i}-c_{i} g_{N}$ for all $0 \leqslant i \leqslant N$. As an analogue of Corollary 2.3 , by induction, we can verify that

$$
w_{i}=\sum_{j=i}^{N} \frac{\widetilde{F}_{i}^{(j)} \tilde{f}_{j}}{q_{j, j-1}}, \quad 1 \leqslant i \leqslant N
$$

From the argument above, it follows immediately that

$$
g_{i}=g_{N}+\sum_{k=i+1}^{N} w_{k}=g_{N}+\sum_{i+1 \leqslant j \leqslant N} \sum_{k \leqslant j \leqslant N} \frac{\widetilde{F}_{k}^{(j)} \tilde{f}_{j}}{q_{j, j-1}}, \quad 0 \leqslant i \leqslant N-1 .
$$

Combining this with the boundary condition $(\Omega g)_{0}=f_{0}$, we finish the proof of the first assertion. The second assertion is derived from the first one immediately.

\section{Uniqueness}

Starting from this section, we handle with the problems for single birth processes, listed at the beginning of the paper. First, we study the uniqueness problem. To do so, we need a sequence $\left(\widetilde{m}_{n}\right)$ (to be used often subsequently) :

$$
\tilde{m}_{0}=\frac{1}{q_{01}}, \quad \tilde{m}_{n}=\frac{1}{q_{n, n+1}}\left(1+\sum_{k=0}^{n-1} \tilde{q}_{n}^{(k)} \tilde{m}_{k}\right), \quad n \geqslant 1 .
$$


By Corollary 2.3, we have

$$
\widetilde{m}_{n}=\sum_{k=0}^{n} \frac{\widetilde{F}_{n}^{(k)}}{q_{k, k+1}}, \quad n \geqslant 0 .
$$

Again, we omit the superscript ${ }^{\sim}$ everywhere in $\tilde{m}, \widetilde{F}$, and $\tilde{q}$ once $c_{i} \equiv 0$. The following criterion is taken from [4, 15, 16].

Proposition 3.1 Corresponding to a given single birth $Q$-matrix $Q=\left(q_{i j}\right)$ (conservative), the process is unique (non-explosive) iff $\sum_{n=0}^{\infty} m_{n}=\infty$.

Proof By [4: Theorems 2.47 and 2.40], the single birth process is unique iff the solution $\left(u_{i}\right)$ to the equation

$$
\left(\lambda+q_{i}\right) u_{i}=\sum_{j \neq i} q_{i j} u_{j}, \quad i \geqslant 0 ; \quad u_{0}=1
$$

is unbounded for some (equivalently for all) $\lambda>0$. Rewrite (3.3) as

$$
\Omega u=Q u-\lambda u=0 ; \quad u_{0}=1 .
$$

Applying Theorem 1.1 to $c_{i} \equiv-\lambda$ and $f_{i} \equiv 0$, we obtain the unique solution:

$$
u_{n}=1+\lambda \sum_{0 \leqslant k \leqslant n-1} \sum_{j=0}^{k} \frac{\widetilde{F}_{k}^{(j)}}{q_{j, j+1}}=1+\lambda \sum_{0 \leqslant k \leqslant n-1} \tilde{m}_{k}, \quad n \geqslant 0 .
$$

Clearly, $u_{n}$ is increasing in $n$ and then is unbounded iff $\sum_{n} \widetilde{m}_{n}=\infty$. Thus, it remains to show that $\sum_{n} \widetilde{m}_{n}=\infty$ iff $\sum_{n} m_{n}=\infty$. Combining $\widetilde{m}_{n}$ with $m_{n}$, it is clear that

$$
\tilde{m}_{n}=\sum_{j=0}^{n} \frac{\widetilde{F}_{n}^{(j)}}{q_{j, j+1}} \downarrow \sum_{k=0}^{n} \frac{F_{n}^{(k)}}{q_{k, k+1}}=m_{n} \quad \text { as } \lambda \downarrow 0,
$$

since

$$
\tilde{q}_{n}^{(k)}=q_{n}^{(k)}+\lambda \quad \downarrow q_{n}^{(k)} \quad \text { as } \lambda \downarrow 0 .
$$

This already shows that the condition $\sum_{n} m_{n}=\infty$ is sufficient. It is nearly necessary since the conclusion does not depend on $\lambda>0$, except there is a jump from $\lambda>0$ to $\lambda=0$. Hopefully, we have thus seen some advantage of Theorem 1.1, even though there is still a distance to prove the necessity.

Actually, there are several ways to prove the equivalence

$$
\sum_{n} \widetilde{m}_{n}=\infty \text { for a fixed } \lambda>0 \Longleftrightarrow \sum_{n} m_{n}=\infty .
$$

From now on, for simplicity, assume that $\lambda=1$. 
(a) Observing that corresponding to the sequence $\left(\tilde{m}_{n}\right)$, the operator is $\Omega=Q-I$ which may be regarded as a bounded perturbation of the original operator $Q$. Since these two operators are zero-exit or not simultaneously, the equivalence above holds.

(b) In the original proof (cf. [4 Proof of Theorem 3.16]), it was proved that $u_{n}$ is unbounded iff $\sum_{n} m_{n}=\infty$. Combining this with what proved above, we obtain the required equivalence.

(c) Here is a more direct proof. The idea comes from [20.

Assume that $\sum_{k=0}^{\infty} \tilde{m}_{k}=\infty$. If $\sum_{k=0}^{\infty} m_{k}<\infty$, then there exists $N_{0}$ large enough such that for all $n \geqslant N_{0}$,

$$
\widetilde{M}_{n}:=\sum_{k=0}^{n} \tilde{m}_{k}>1 \quad \text { and } \quad K:=2 \sum_{k=N_{0}+1}^{\infty} m_{k}<1 .
$$

We now prove that for each $n>N_{0}$,

$$
\tilde{m}_{k} \leqslant 2 m_{k} \widetilde{M}_{n-1}, \quad 0 \leqslant k \leqslant n .
$$

Since $\widetilde{m}_{0}=m_{0}$ and $\widetilde{M}_{n-1}>1$ (due to the fact that $n-1 \geqslant N_{0}$ ), (3.4) holds in the case of $k=0$. Assume that (3.4) holds up to $k=\ell-1<n$. Then,

$$
\begin{aligned}
\tilde{m}_{\ell} & =\frac{1}{q_{\ell, \ell+1}}\left(1+\sum_{k=0}^{\ell-1} q_{\ell}^{(k)} \tilde{m}_{k}+\sum_{k=0}^{\ell-1} \widetilde{m}_{k}\right) \quad(\text { since } \lambda=1) \\
& \leqslant \frac{1}{q_{\ell, \ell+1}}\left(1+\sum_{k=0}^{\ell-1} q_{\ell}^{(k)} 2 m_{k} \widetilde{M}_{n-1}+\widetilde{M}_{\ell-1}\right) \quad \text { (by assumption) } \\
& \leqslant \frac{1}{q_{\ell, \ell+1}}\left(1+\sum_{k=0}^{\ell-1} q_{\ell}^{(k)} m_{k}\right) 2 \widetilde{M}_{n-1} \\
& =2 m_{\ell} \widetilde{M}_{n-1} .
\end{aligned}
$$

So (3.4) holds when $k=\ell$. By induction, we know that (3.4) holds for every $k: 0 \leqslant k \leqslant n$. Now, for each $n>N_{0}$, we have

$$
\widetilde{M}_{n}=\widetilde{M}_{N_{0}}+\sum_{k=N_{0}+1}^{n} \widetilde{m}_{k} \leqslant \widetilde{M}_{N_{0}}+\sum_{k=N_{0}+1}^{n} 2 m_{k} \widetilde{M}_{n-1} \leqslant \widetilde{M}_{N_{0}}+K \widetilde{M}_{n-1} .
$$

Furthermore, we have

$$
\begin{aligned}
\widetilde{M}_{n} & \leqslant \widetilde{M}_{N_{0}}\left(1+K+\cdots+K^{n-N_{0}-1}\right)+K^{n-N_{0}} \widetilde{M}_{N_{0}} \\
& =\frac{\widetilde{M}_{N_{0}}\left(1-K^{n-N_{0}}\right)}{1-K}+K^{n-N_{0}} \widetilde{M}_{N_{0}} .
\end{aligned}
$$

Thus, as $n \rightarrow \infty$, we would have $\infty \leqslant \widetilde{M}_{N_{0}} /(1-K)$ which is a contradiction. Hence, once $\sum_{k=0}^{\infty} \widetilde{m}_{k}=\infty$, we should also have $\sum_{k=0}^{\infty} m_{k}=\infty$. 
We have therefore completed the proof of the equivalence mentioned above.

To conclude this section, we mention that the uniqueness problem for the single birth $Q$-matrix with absorbing set $H=\{0,1, \ldots, N\}(N<\infty)$ can be dealt with by the same approach. Refer to [4, Theorem 3.16] and [14].

\section{Recurrence and extinction/return probability}

For the recurrence, the following criterion is taken from [4: Theorem 4.52 (1)] and [15].

Proposition 4.1 Assume the single birth $Q$-matrix $Q=\left(q_{i j}\right)$ is non-explosive and irreducible. Then the process is recurrent iff $\sum_{n=0}^{\infty} F_{n}^{(0)}=\infty$, where $\left(F_{n}^{(i)}\right)$ was defined in (1.1) by setting $c_{i} \equiv 0$.

Proof By [4: Lemma 4.51], we know that the single birth process is recurrent iff the equation

$$
x_{i}=\sum_{k \neq 0} \Pi_{i k} x_{k}, \quad 0 \leqslant x_{i} \leqslant 1, \quad i \geqslant 0
$$

has only zero solution, where $\Pi_{i k}=\left(1-\delta_{i k}\right) q_{i k} / q_{i}$. It is easily seen that equation (4.1) has a non-trivial solution iff the equation

$$
x_{i}=\sum_{k \neq 0} \Pi_{i k} x_{k}, \quad i \geqslant 0 ; \quad x_{0}=1
$$

has a nonnegative bounded solution. The following fact will be used several times below:

$$
x_{i}=\sum_{k \neq i, i_{0}} \frac{q_{i k}}{q_{i}-\lambda} x_{k}+\frac{\gamma_{i}}{q_{i}-\lambda} \Longleftrightarrow(Q x)_{i}+\lambda x_{i}=q_{i i_{0}}\left(1-\delta_{i i_{0}}\right) x_{i_{0}}-\gamma_{i},
$$

where $\lambda \in \mathbb{R}$ satisfying some suitable condition. Certainly, here we preassume that $x_{i} \in \mathbb{R}$ for every $i \in E$. By using this fact with $\lambda=0$ and $i_{0}=0$, we can rewrite the previous equation as

$$
(Q x)_{0}=0, \quad(Q x)_{i}=q_{i 0}, \quad i \geqslant 1 ; \quad x_{0}=1 .
$$

Applying Theorem 1.1 to $c_{i} \equiv 0$ and $f_{i}=q_{i 0}\left(1-\delta_{i 0}\right)$, we obtain the unique solution as follows

$$
x_{0}=1, \quad x_{n}=1+\sum_{k=1}^{n-1} \sum_{j=1}^{k} \frac{F_{k}^{(j)} q_{j 0}}{q_{j, j+1}}=1+\sum_{k=1}^{n-1} \sum_{j=1}^{k} \frac{F_{k}^{(j)} q_{j}^{(0)}}{q_{j, j+1}}, \quad n \geqslant 1 .
$$


By (2.7), it follows that

$$
x_{n}=1+\sum_{k=1}^{n-1} F_{k}^{(0)}=\sum_{k=0}^{n-1} F_{k}^{(0)}, \quad n \geqslant 1 .
$$

Clearly, $\left(x_{n}\right)$ is bounded iff $\sum_{k=0}^{\infty} F_{k}^{(0)}<\infty$. In other words, equation (4.1) has only a trivial solution iff $\sum_{k=0}^{\infty} F_{k}^{(0)}=\infty$. The assertion is now proven.

\section{Extinction/return probability}

For the remainder of this section, we study the extinction probability. Here the extinction time $\tau_{0}$ is the first hitting time of the state 0 . Thus, this topic is actually a refinement of what studied in the last proposition, in which we pay attention only on the result either $\mathbb{P}_{n}\left[\tau_{0}<\infty\right]=1$ or $<1$ rather than its distribution. We will come back this point after the proof of the next proposition. For the extinction problem, the rates $q_{0 j}(j \neq 0)$ play no rule, so one may assume the state 0 to be an absorbing state. In other words, we may reduce the state space from $E$ to $E_{1}:=\{1,2, \ldots\}$, and regard the rate $q_{i 0}(i \neq 0)$ as a killing from $i$. Then we need to redefine the sequences $\left(\tilde{q}_{n}^{(k)}\right)$ and $\left(\widetilde{F}_{n}^{(k)}\right)$ starting from 1 but not 0 . However, for our convenience, we prefer to keep the notation $E,\left(\tilde{q}_{n}^{(k)}\right),\left(\widetilde{F}_{n}^{(k)}\right)$ and so on. For this, it is better to use the return time $\sigma_{0}$ instead of the hitting time $\tau_{0}$. In the case that the state 0 is really an absorbing one, we can add a positive rate $q_{01}$ and assume that the enlarged process becomes irreducible. Then, the solution of $\mathbb{P}_{n}\left[\sigma_{0}<\infty\right]$ restricted on $E_{1}$ gives us the answer of $\mathbb{P}_{n}\left[\tau_{0}<\infty\right]$ on $E_{1}$ (as a trivial application of the localization theorem [9, Theorem 3.4.1] or [4 Theorem 2.13]), so we can return to our original problem.

We remark that in the context of denumerable Markov processes, the topic of this section and much more problems were well studied in [9. Chapter IX]. In the present special case, for the single birth processes, the problem was studied in [1. Chapter 9] or [2], using a different technique.

Proposition 4.2 Let the single birth $Q$-matrix $Q=\left(q_{i j}\right)$ be non-explosive and irreducible. Then the return/extinction probability is as follows:

$$
\mathbb{P}_{0}\left(\sigma_{0}<\infty\right)=\frac{\sum_{k=1}^{\infty} F_{k}^{(0)}}{\sum_{k=0}^{\infty} F_{k}^{(0)}}, \quad \mathbb{P}_{n}\left(\sigma_{0}<\infty\right)=\frac{\sum_{k=n}^{\infty} F_{k}^{(0)}}{\sum_{k=0}^{\infty} F_{k}^{(0)}}, \quad n \geqslant 1 .
$$

Furthermore, $\mathbb{P}_{n}\left(\sigma_{0}<\infty\right)=1$ for all $n \geqslant 0$ iff $\mathbb{P}_{0}\left(\sigma_{0}<\infty\right)=1$, equivalently iff $\sum_{n=0}^{\infty} F_{n}^{(0)}=\infty$.

Proof By [4 Lemma 4.46] with $H=\{0\},\left(\mathbb{P}_{i}\left(\sigma_{0}<\infty\right): i \in E\right)$ is the minimal nonnegative solution to the equation

$$
x_{i}=\sum_{k \neq 0, i} \frac{q_{i k}}{q_{i}} x_{k}+\frac{q_{i 0}}{q_{i}}\left(1-\delta_{i 0}\right), \quad i \in E .
$$


The study on recurrence usually starts from here, the lemma [4: Lemma 4.51] used in the last proof simplifies our study on the recurrence problem, as we have just seen above. By (4.2), the last equation is equivalent to

$$
(Q x)_{i}=q_{i 0}\left(1-\delta_{i 0}\right)\left(x_{0}-1\right), \quad i \geqslant 0 .
$$

Applying Theorem 1.1 to $c_{i} \equiv 0$ and $f_{i}=q_{i 0}\left(1-\delta_{i 0}\right)\left(x_{0}-1\right)$, we obtain the solution to the last equation:

$$
\begin{aligned}
x_{n} & =x_{0}+\sum_{0 \leqslant k \leqslant n-1} \sum_{0 \leqslant j \leqslant k} \frac{F_{k}^{(j)}}{q_{j, j+1}} q_{j 0}\left(1-\delta_{j 0}\right)\left(x_{0}-1\right) \\
& =x_{0}\left\{1+\sum_{1 \leqslant k \leqslant n-1} \sum_{1 \leqslant j \leqslant k} \frac{F_{k}^{(j)}}{q_{j, j+1}} q_{j}^{(0)}\right\}-\sum_{1 \leqslant k \leqslant n-1} \sum_{1 \leqslant j \leqslant k} \frac{F_{k}^{(j)}}{q_{j, j+1}} q_{j}^{(0)} \\
& \left.=x_{0}\left(1+\sum_{1 \leqslant k \leqslant n-1} F_{k}^{(0)}\right)-\sum_{1 \leqslant k \leqslant n-1} F_{k}^{(0)}, \quad n \geqslant 0 \quad \text { (by (2.7) }\right) .
\end{aligned}
$$

Because $x_{n}>0$, it follows that

$$
x_{0} \geqslant \sup _{n \geqslant 1} \frac{\sum_{k=1}^{n-1} F_{k}^{(0)}}{\sum_{k=0}^{n-1} F_{k}^{(0)}}=\sup _{n \geqslant 1} \frac{\sum_{k=0}^{n-1} F_{k}^{(0)}-1}{\sum_{k=0}^{n-1} F_{k}^{(0)}}=1-\frac{1}{\sum_{k=0}^{\infty} F_{k}^{(0)}} .
$$

From here, we obtain the minimal nonnegative solution:

$$
x_{0}^{*}=1-\frac{1}{\sum_{k=0}^{\infty} F_{k}^{(0)}}, \quad x_{n}^{*}=1-\frac{\sum_{k=0}^{n-1} F_{k}^{(0)}}{\sum_{k=0}^{\infty} F_{k}^{(0)}}, \quad n \geqslant 1 .
$$

We have thus proved the first assertion. The second one is obvious.

Rewrite the solution just obtained as follows.

$$
1-x_{0}^{*}=\frac{1}{\sum_{k=0}^{\infty} F_{k}^{(0)}}, \quad 1-x_{n}^{*}=\frac{\sum_{k=0}^{n-1} F_{k}^{(0)}}{\sum_{k=0}^{\infty} F_{k}^{(0)}}, \quad n \geqslant 1 .
$$

Renormalize them so that the initial value becomes 1 :

$$
x_{0}=1, \quad x_{n}=\sum_{k=0}^{n-1} F_{k}^{(0)}, \quad n \geqslant 1
$$

which is what we obtained in the last proof. We have thus seen the relation between the last two propositions.

The study on the Laplace transform of extinction/return time is delayed to Section 7 (Proposition 7.3 which is based on Lemma 7.1). 


\section{Ergodicity, strong ergodicity, and the first moment of return time}

Let $E=\mathbb{Z}_{+}$and $H \subset E, H \neq \varnothing, E$. Define $\sigma_{H}=\inf \left\{t \geqslant \eta_{1}: X(t) \in H\right\}$, where $\eta_{1}$ is the first jump of the process. When $H$ is a singleton, $H=\{0\}$, for instance, denote $\sigma_{\{0\}}$ by $\sigma_{0}$ for simplicity. We now consider the first moment of the return time $\sigma_{0}$. To do so, we introduce the following lemma (cf. 9. Lemma 9.4.1]).

Lemma 5.1 Let $\left(q_{i j}\right)$ be irreducible and assume that its $Q$-process is recurrent. Then $\left(x_{i}^{*}:=\mathbb{E}_{i} \sigma_{H}: i \in E\right)$ is the minimal nonnegative solution (may be infinite) to the equation

$$
x_{i}=\frac{1}{q_{i}} \sum_{k \notin H \cup\{i\}} q_{i k} x_{k}+\frac{1}{q_{i}}, \quad i \in E,
$$

where $1 \cdot \infty=\infty$ and $0 \cdot \infty=0$ by convention.

Proof Let $\left(y_{i}^{*}: i \in E\right)$ be the minimal nonnegative solution to the equation

$$
y_{i}=\frac{1}{q_{i}} \sum_{k \notin H \cup\{i\}} q_{i k} y_{k}+\frac{1}{q_{i}}, \quad i \in E .
$$

By assumption and [4 Lemma 4.46], the quantity $f_{i H}$ defined there is equal to 1 for every $i \in E$. Then, $\left(y_{i}^{*}: i \in E\right)$ coincides with $\left(e_{i H}(0): i \in E\right)$ used in 4 . Lemma 4.48]. Note that $e_{i H}(0)=\int_{0}^{\infty} \mathbb{P}_{i}\left(\sigma_{H}>t\right) \mathrm{d} t=\mathbb{E}_{i} \sigma_{H}$. The assertion now follows immediately.

In what follows, we use often another sequence $\left(\tilde{d}_{n}\right)$ similar to $\left(\widetilde{m}_{n}\right)$ having different initial value:

$$
\tilde{d}_{0}=0, \quad \tilde{d}_{n}=\frac{1}{q_{n, n+1}}\left(1+\sum_{k=0}^{n-1} \tilde{q}_{n}^{(k)} \tilde{d}_{k}\right), \quad n \geqslant 1,
$$

where $\tilde{q}_{n}^{(k)}$ is defined in (1.2). By Corollary 2.3, we have

$$
\tilde{d}_{n}=\sum_{1 \leqslant j \leqslant n} \frac{\tilde{F}_{n}^{(j)}}{q_{j, j+1}}, \quad n \geqslant 0
$$

which is very much the same as (3.2). Again, we omit the superscript everywhere in $\left(\tilde{d}_{n}\right)$ once $c_{i} \equiv 0$. Note that if we rewrite

$$
\begin{gathered}
\tilde{d}_{n}=\frac{1}{q_{n, n+1}}\left(1+\sum_{1 \leqslant k \leqslant n-1} \tilde{q}_{n}^{(k)} \tilde{d}_{k}\right), \quad n \geqslant 1, \\
\widetilde{F}_{n}^{(0)}=\frac{1}{q_{n, n+1}}\left(\tilde{q}_{n}^{(0)}+\sum_{1 \leqslant k \leqslant n-1} \tilde{q}_{n}^{(k)} \widetilde{F}_{k}^{(0)}\right), \quad n \geqslant 1,
\end{gathered}
$$


then it is clear that the sequences $\left(\tilde{d}_{n}\right)_{n \geqslant 1}$ and $\left(\widetilde{F}_{n}^{(0)}\right)_{n \geqslant 1}$ are also quite close each other.

The main result in this section is as follows. Refer to [4 Theorem $4.52(2)$ ], [1. Proposition 2.4], and [15, 17, 18].

Proposition 5.2 Assume that the single birth $Q$-matrix $Q=\left(q_{i j}\right)$ is irreducible and corresponding process is recurrent. Then

$$
\mathbb{E}_{0} \sigma_{0}=\frac{1}{q_{01}}+d, \quad \mathbb{E}_{n} \sigma_{0}=\sum_{k=0}^{n-1}\left(F_{k}^{(0)} d-d_{k}\right), \quad n \geqslant 1,
$$

where

$$
d=\varlimsup_{k \rightarrow \infty} \frac{\sum_{n=0}^{k} d_{n}}{\sum_{n=0}^{k} F_{n}^{(0)}}=\lim _{n \rightarrow \infty} \frac{d_{n}}{F_{n}^{(0)}} \text { if the limit exists. }
$$

Furthermore, the process is ergodic (i.e. positive recurrent) iff $d<\infty$; and it is strongly ergodic iff $\sup _{k \in E} \sum_{n=0}^{k}\left(F_{n}^{(0)} d-d_{n}\right)<\infty$. Actually, for the last conclusion, the recurrence assumption can be replaced by the uniqueness one.

Proof Let $H=\{0\}$. By Lemma [5.1, $\left(\mathbb{E}_{i} \sigma_{0}: i \in E\right)$ is the minimal nonnegative solution $\left(x_{i}^{*}\right)$ to the equation

$$
x_{i}=\frac{1}{q_{i}} \sum_{k \notin\{0, i\}} q_{i k} x_{k}+\frac{1}{q_{i}}, \quad i \in E .
$$

Suppose for a moment that $x_{i}^{*}<\infty$ first for some $i \in E$ and then for all $i$ by irreducibility. Next, let $\left(x_{i}\right)$ be a (finite) solution to (5.3). Then, by (4.2), we have

$$
(Q x)_{i}=q_{i 0} x_{0}-1, \quad i \geqslant 1 ; \quad(Q x)_{0}=-1 .
$$

Applying Theorem 1.1 to $c=0$ and $f_{i}=q_{i 0}\left(1-\delta_{i 0}\right) x_{0}-1(i \geqslant 0)$, we obtain the solution to the last equation:

$x_{n}=x_{0}+\sum_{k=0}^{n-1} \sum_{j=0}^{k} \frac{F_{k}^{(j)} f_{j}}{q_{j, j+1}}=x_{0}\left(1+\sum_{k=1}^{n-1} \sum_{j=1}^{k} \frac{F_{k}^{(j)} q_{j 0}}{q_{j, j+1}}\right)-\sum_{k=0}^{n-1} \sum_{j=0}^{k} \frac{F_{k}^{(j)}}{q_{j, j+1}}, \quad n \geqslant 1$.

By (2.7) and (5.2), we obtain

$$
x_{n}=x_{0} \sum_{k=0}^{n-1} F_{k}^{(0)}-\sum_{k=0}^{n-1}\left(\frac{F_{k}^{(0)}}{q_{01}}+d_{k}\right)=\sum_{k=0}^{n-1}\left[F_{k}^{(0)}\left(x_{0}-\frac{1}{q_{01}}\right)-d_{k}\right], \quad n \geqslant 1 .
$$

Since $x_{n}>0$, it follows that

$$
x_{0} \sum_{k=0}^{n-1} F_{k}^{(0)}>\sum_{k=0}^{n-1}\left(\frac{F_{k}^{(0)}}{q_{01}}+d_{k}\right), \quad n \geqslant 1
$$


This gives us

$$
x_{0} \geqslant \sup _{n \geqslant 1} \frac{\sum_{k=0}^{n-1}\left(F_{k}^{(0)} / q_{01}+d_{k}\right)}{\sum_{k=0}^{n-1} F_{k}^{(0)}}=\frac{1}{q_{01}}+\sup _{n \geqslant 1} \frac{\sum_{k=0}^{n-1} d_{k}}{\sum_{k=0}^{n-1} F_{k}^{(0)}} .
$$

Now, the minimal property implies that

$$
x_{0}^{*}=\frac{1}{q_{01}}+\sup _{n \geqslant 1} \frac{\sum_{k=0}^{n-1} d_{k}}{\sum_{k=0}^{n-1} F_{k}^{(0)}}
$$

and then

$$
x_{n}^{*}=\sum_{k=0}^{n-1}\left(F_{k}^{(0)} \sup _{n \geqslant 1} \frac{\sum_{j=0}^{n-1} d_{j}}{\sum_{j=0}^{n-1} F_{j}^{(0)}}-d_{k}\right), \quad n \geqslant 1
$$

gives us the solution $\left(\mathbb{E}_{i} \sigma_{0}: i \in E\right)$. We claim that the supremum in the last line has to achieved at infinity. Otherwise, if it is achieved at some finite $n_{0}$ :

$$
\frac{\sum_{j=0}^{n_{0}-1} d_{j}}{\sum_{j=0}^{n_{0}-1} F_{j}^{(0)}}=\sup _{n \geqslant 1} \frac{\sum_{j=0}^{n-1} d_{j}}{\sum_{j=0}^{n-1} F_{j}^{(0)}} \text {. }
$$

Then

$$
x_{0}^{*}=\frac{1}{q_{01}}+\frac{\sum_{j=0}^{n_{0}-1} d_{j}}{\sum_{j=0}^{n_{0}-1} F_{j}^{(0)}}
$$

and furthermore, $x_{n_{0}}^{*}=0$ which is a contradiction with $x_{i}^{*}=\mathbb{E}_{i} \sigma_{0}>0$. Therefore,

$$
\sup _{n \geqslant 1} \frac{\sum_{j=0}^{n-1} d_{j}}{\sum_{j=0}^{n-1} F_{j}^{(0)}}=\varlimsup_{n \rightarrow \infty} \frac{\sum_{j=0}^{n} d_{j}}{\sum_{j=0}^{n} F_{j}^{(0)}}=: d
$$

as required. The next limit in the expression of $d$ is an application of Stolz's Theorem. Now $d<\infty$ since $x_{0}^{*}<\infty$ by assumption. To remove the finiteness assumption of $\left(x_{i}^{*}\right)$, we claim that the expressions in the first assertion for $\mathbb{E}_{n} \sigma_{0}\left(=x_{n}^{*}\right)$ still hold even $x_{i}^{*}=\infty$, since then we must have $d=\infty$. If otherwise, $d<\infty$, then by the last assertion of Theorem 1.1 and (4.2), we would obtain a finite solution to (5.3), which deduces a contradiction to the assumption $x_{i}^{*}=\infty$ by the comparison theorem for the nonnegative solutions (cf. 4. Theorem 2.6]). We have thus proved the first assertion.

Let us remark that the trick used above replacing $\sup _{n \geqslant 1}$ by $\varlimsup_{n \rightarrow \infty}$ was missed in the previous publications. This trick and the one assuming the finiteness of $\left(x_{i}^{*}\right)$, will be used several times below but we may not mention it time by time.

Finally, by [4. Theorem 4.44], the single process is ergodic iff $\mathbb{E}_{0} \sigma_{0}<\infty$ which is now equivalent to $d<\infty$. By the same cited theorem, the process is strongly ergodic iff $\sup _{i \in E} \mathbb{E}_{i} \sigma_{0}<\infty$, equivalently, $\sup _{n \in E} \sum_{k=0}^{n}\left(F_{k}^{(0)} d-d_{k}\right)<$ $\infty$ which follows from the first assertion. As mentioned in the proof of the 
cited book, for ergodicity, the uniqueness assumption is enough instead of the recurrence one. The proof is now finished.

\section{Polynomial moments of hitting time and life time}

\section{Polynomial moments of hitting time}

We have just studied the first moment of the time of first hitting/return 0 in the last section. Now we study the higher-order moments of the first hitting time.

Fix $i_{0} \geqslant 0$. Recall that $\sigma_{i_{0}}$ is the time of first return to $i_{0}$ after the first jump. For its higher-moments, we have the following result (cf. [19, 21]).

Proposition 6.1 Assume that the single birth $Q$-matrix $Q=\left(q_{i j}\right)$ is irreducible and the corresponding process is $(\ell-1)$-ergodic $(\ell \geqslant 1)$, i.e. $\mathbb{E}_{i} \sigma_{i_{0}}^{\ell-1}<\infty$ for every $i \geqslant 0$. When $\ell=1$, assume additionally that the process is unique. Then we have

$$
\mathbb{E}_{n} \sigma_{i_{0}}^{\ell}= \begin{cases}\ell \sum_{n \leqslant k \leqslant i_{0}-1} v_{k}^{(\ell)}+\left[1-\sum_{n \leqslant k \leqslant i_{0}-1} u_{k}\right] \mathbb{E}_{i_{0}} \sigma_{i_{0}}^{\ell}, & 0 \leqslant n \leqslant i_{0} \\ -\ell \sum_{i_{0} \leqslant k \leqslant n-1} v_{k}^{(\ell)}+\left[1+\sum_{i_{0} \leqslant k \leqslant n-1} u_{k}\right] \mathbb{E}_{i_{0}} \sigma_{i_{0}}^{\ell}, & n>i_{0} ;\end{cases}
$$

where

$$
\begin{aligned}
& u_{k}= \begin{cases}\sum_{j=i_{0}-1}^{k} q_{j, j+1}{ }^{-1} F_{k}^{(j)} q_{j i_{0}}\left(1-\delta_{j i_{0}}\right), & k \geqslant i_{0}, \\
1, & k=i_{0}-1, \\
0, & 0 \leqslant k \leqslant i_{0}-2\end{cases} \\
& v_{k}^{(\ell)}=\sum_{j=0}^{k} \frac{F_{k}^{(j)}}{q_{j, j+1}} \mathbb{E}_{j} \sigma_{i_{0}}^{\ell-1}, \quad k \geqslant 0, \\
& \mathbb{E}_{i_{0}} \sigma_{i_{0}}^{\ell}=\ell \varlimsup_{n \rightarrow \infty}\left(\sum_{i_{0} \leqslant k \leqslant n} v_{k}^{(\ell)}\right)\left[1+\sum_{i_{0} \leqslant k \leqslant n} u_{k}\right]^{-1} \\
& =\ell \lim _{n \rightarrow \infty} \frac{v_{n}^{(\ell)}}{u_{n}} \text { if the limit exists. }
\end{aligned}
$$

Proof By 9, Theorem 9.3.3] (cf. 4, Proposition 4.56], or [10, Theorem 3.1]), $\left(y_{i}^{*}:=\mathbb{E}_{i} \sigma_{i_{0}}^{\ell}: i \in E\right)$ is the the minimal nonnegative solution to the following equation:

$$
y_{i}=\sum_{k \neq i, i_{0}} \frac{1}{q_{i}} q_{i k} y_{k}+\frac{\ell}{q_{i}} \mathbb{E}_{i} \sigma_{i_{0}}^{\ell-1}, \quad i \in E .
$$

As remarked in the last section, we may assume that $y_{i}^{*}<\infty$ for every $i \in E$. Then, by (4.2), we obtain the Poisson equation:

$$
(Q y)_{i}=q_{i i_{0}}\left(1-\delta_{i i_{0}}\right) y_{i_{0}}-\ell \mathbb{E}_{i} \sigma_{i_{0}}^{\ell-1}, \quad i \in E .
$$


Applying Theorem 1.1 to $c=0$ and $f_{i}=q_{i i_{0}}\left(1-\delta_{i i_{0}}\right) y_{i_{0}}-\ell \mathbb{E}_{i} \sigma_{i_{0}}^{\ell-1}$, it follows that the solution to the last equation is as follows:

$$
y_{n}=y_{0}+\sum_{0 \leqslant k \leqslant n-1} \sum_{j=0}^{k} \frac{F_{k}^{(j)} f_{j}}{q_{j, j+1}}=y_{0}+y_{i_{0}} \sum_{0 \leqslant k \leqslant n-1} u_{k}-\ell \sum_{0 \leqslant k \leqslant n-1} v_{k}^{(\ell)}, \quad n \geqslant 0 .
$$

Here in the summation of $u_{k}$, we have used the character of single birth: $q_{j i_{0}}(1-$ $\left.\delta_{j i_{0}}\right)>0$ only if either $j=i_{0}-1$ or $j \geqslant i_{0}+1$. In particular, by setting $n=i_{0}$, it follows that

$$
y_{0}=\ell \sum_{0 \leqslant k \leqslant i_{0}-1} v_{k}^{(\ell)}+y_{i_{0}}\left(1-\sum_{0 \leqslant k \leqslant i_{0}-1} u_{k}\right) .
$$

Return to the original $y_{n}$, we get

$$
\begin{aligned}
y_{n} & =\ell\left[\sum_{0 \leqslant k \leqslant i_{0}-1} v_{k}^{(\ell)}-\sum_{0 \leqslant k \leqslant n-1} v_{k}^{(\ell)}\right]+y_{i_{0}}\left[1-\sum_{0 \leqslant k \leqslant i_{0}-1} u_{k}+\sum_{0 \leqslant k \leqslant n-1} u_{k}\right] \\
& = \begin{cases}-\ell \sum_{i_{0} \leqslant k \leqslant n-1} v_{k}^{(\ell)}+y_{i_{0}}\left[1+\sum_{i_{0} \leqslant k \leqslant n-1} u_{k}\right], \quad n \geqslant i_{0}+1 \\
\ell \sum_{n \leqslant k \leqslant i_{0}-1} v_{k}^{(\ell)}+y_{i_{0}}\left[1-\sum_{n \leqslant k \leqslant i_{0}-1} u_{k}\right], & n \leqslant i_{0} .\end{cases}
\end{aligned}
$$

When $n \leqslant i_{0}$, since $\sum_{k \leqslant i_{0}-1} u_{k} \leqslant 1$ by definition of $\left(u_{k}\right)$, it is clear that $y_{n}>0$. When $n \geqslant i_{0}+1$, for $y_{n}>0$, one requires the condition

$$
y_{i_{0}}>\frac{\ell \sum_{i_{0} \leqslant k \leqslant n-1} v_{k}^{(\ell)}}{1+\sum_{i_{0} \leqslant k \leqslant n-1} u_{k}}
$$

and then

$$
y_{i_{0}} \geqslant \sup _{n \geqslant i_{0}+1} \frac{\ell \sum_{i_{0} \leqslant k \leqslant n-1} v_{k}^{(\ell)}}{1+\sum_{i_{0} \leqslant k \leqslant n-1} u_{k}} .
$$

By a reason explained in the last section, this leads to

$$
y_{i_{0}}^{*}=\ell \varlimsup_{n \rightarrow \infty} \frac{\sum_{i_{0} \leqslant k \leqslant n} v_{k}^{(\ell)}}{1+\sum_{i_{0} \leqslant k \leqslant n} u_{k}}
$$

which gives us $\mathbb{E}_{i_{0}} \sigma_{i_{0}}^{\ell}$. Combining it with (6.1), we obtain the required assertion. The limit in $\mathbb{E}_{i_{0}} \sigma_{i_{0}}^{\ell}$ is again an application of Stolz's Theorem since $\sum_{k} u_{k}=\infty$ by the recurrence of the process. To see the last assertion, define a single birth process on $\left\{i_{0}, i_{0}+1, \ldots\right\}$ (regarding the set $\left\{0,1, \ldots, i_{0}\right\}$ as a single state) with rates

$$
\bar{q}_{i j}= \begin{cases}q_{i j} & \text { if } j \geqslant i_{0}+1 \\ \sum_{k \leqslant i_{0}} q_{i k} & \text { if } j=i_{0}, \quad i \geqslant i_{0} .\end{cases}
$$


Then $\left(\bar{q}_{i j}\right)$ is irreducible and recurrent because so is $\left(q_{i j}\right)$. Next, as in (1.1), we can define a sequence $\left(\bar{F}_{k}^{(j)}\right)$ on $\left\{i_{0}, i_{0}+1, \ldots\right\}$. By induction, it is easy to check that $\bar{F}_{k}^{(j)}=\widetilde{F}_{k}^{(j)}$ for every $k \geqslant j \geqslant i_{0}$. Hence we have

$$
\sum_{k} \bar{F}_{k}^{\left(i_{0}\right)}=\sum_{k} \widetilde{F}_{k}^{\left(i_{0}\right)}=\infty
$$

by Proposition 4.1. It should be now easy to see that $\sum_{k} u_{k}=\infty$ as claimed. $\square$

\section{Polynomial moments of life time}

Recall that $\tau_{n}$ is the time of first hitting the state $n$. If we start from $i \leqslant n-1$, then $\tau_{n}$ coincides with the time of fist hitting the set $\{n, n+1, \ldots\}$. For the remainder of this section, we are going to study the time $\tau_{\infty}:=\lim _{n \rightarrow \infty} \tau_{n}$. Next, because $\tau_{\infty}$ is actually equal to the life time $\eta:=\lim _{n \rightarrow \infty} \eta_{n}$ almost everywhere, where $\left\{\eta_{n}\right\}$ are the successive jumping times:

$$
\eta_{0} \equiv 0, \quad \eta_{n}=\inf \left\{t \geqslant \eta_{n-1}: X(t) \neq X\left(\eta_{n-1}\right)\right\}, \quad n \geqslant 1,
$$

therefore, $\tau_{\infty}=\infty$ a.e. if the single birth $Q$-matrix is non-explosive. Thus, the study on the moments of $\tau_{\infty}$ is meaningful only for explosive single birth $Q$-matrix. The next result is taken from [21].

Proposition 6.2 Let the single birth $Q$-matrix $Q=\left(q_{i j}\right)$ be irreducible and explosive (i.e. $\sum_{n} m_{n}<\infty$ by Proposition 3.1). Assume that the minimal process has finite $(\ell-1)$-th moments of $\tau_{\infty}$ for some integer $\ell \geqslant 1$ (i.e. $E_{i} \tau_{\infty}^{\ell-1}<$ $\infty$ for all $i \geqslant 0)$. Then

$$
\mathbb{E}_{n} \tau_{\infty}^{\ell}=\ell \sum_{k \geqslant n} \bar{m}_{k}^{(\ell)}, \quad n \geqslant 0
$$

where

$$
\bar{m}_{n}^{(\ell)}=\frac{1}{q_{n, n+1}}\left[\mathbb{E}_{n} \tau_{\infty}^{\ell-1}+\sum_{0 \leqslant k \leqslant n-1} q_{n}^{(k)} \bar{m}_{k}^{(\ell)}\right]=\sum_{j=0}^{n} \frac{F_{n}^{(j)} \mathbb{E}_{j} \tau_{\infty}^{\ell-1}}{q_{j, j+1}}, \quad n \geqslant 0 .
$$

Proof The last equality of $\bar{m}_{n}^{(\ell)}$ comes from Corollary 2.3, By [4: Proposition 4.56] or [11, we know that $\left(\mathbb{E}_{i} \tau_{\infty}^{\ell}: i \in E\right)$ is the the minimal nonnegative solution $\left(y_{i}^{*}: i \in E\right)$ to the following equation:

$$
y_{i}=\sum_{k \neq i} \frac{1}{q_{i}} q_{i k} y_{k}+\frac{\ell}{q_{i}} \mathbb{E}_{i} \tau_{\infty}^{\ell-1}, \quad i \in E .
$$

That is,

$$
(Q y)_{i}=-\ell \mathbb{E}_{i} \tau_{\infty}^{\ell-1}, \quad i \in E
$$


Applying Theorem 1.1 to $c=0$ and $f_{i}=-\ell \mathbb{E}_{i} \tau_{\infty}^{\ell-1}(i \geqslant 0)$, it follows that the solution to the last equation can be expressed as

$$
y_{n}=y_{0}-\ell \sum_{k=0}^{n-1} \sum_{j=0}^{k} \frac{F_{k}^{(j)} \mathbb{E}_{j} \tau_{\infty}^{\ell-1}}{q_{j, j+1}}, \quad n \geqslant 1 .
$$

Hence

$$
y_{n}=y_{0}-\ell \sum_{k=0}^{n-1} \bar{m}_{k}^{(\ell)}, \quad n \geqslant 1
$$

By the nonnegative and minimal properties, it follows that

$$
y_{0}^{*}=\sup _{n \geqslant 1}\left(\ell \sum_{k=0}^{n-1} \bar{m}_{k}^{(\ell)}\right)=\ell \sum_{k=0}^{\infty} \bar{m}_{k}^{(\ell)}, \quad y_{n}^{*}=\ell \sum_{k=n}^{\infty} \bar{m}_{k}^{(\ell)}, \quad n \geqslant 1 .
$$

Hence, we obtain

$$
\mathbb{E}_{n} \tau_{\infty}^{\ell}=\ell \sum_{k \geqslant n} \bar{m}_{k}^{(\ell)}, \quad n \geqslant 0
$$

which is the required assertion.

\section{Exponential ergodicity and Laplace transform of return time}

\section{Exponential moments of return time and exponential ergodicity}

In this section, we consider the exponential moments of return time. At first, we introduce the following lemma for general $Q$-matrices.

Lemma 7.1 Let $\left(q_{i j}\right)$ be irreducible and assume that its $Q$-process is recurrent. Next, let $\lambda \in \mathbb{R}, \lambda<q_{i}$ for every $i \in E$. Then for fixed $H \subset E, H \neq \varnothing, E$, $\left(\mathbb{E}_{i} \exp \left(\lambda \sigma_{H}\right): i \in E\right)$ is the minimal solution to the equation

$$
x_{i}=\frac{1}{q_{i}-\lambda} \sum_{k \notin H \cup\{i\}} q_{i k} x_{k}+\frac{1}{q_{i}-\lambda} \sum_{k \in H \backslash\{i\}} q_{i k}, \quad i \in E .
$$

Proof Let $\left(y_{i}^{*}: i \in E\right)$ be the minimal nonnegative solution to the equation

$$
y_{i}=\frac{1}{q_{i}-\lambda} \sum_{k \notin H \cup\{i\}} q_{i k} y_{k}+\frac{1}{q_{i}-\lambda}, \quad i \in E .
$$

By the recurrent assumption and [4 Lemma 4.46], the quantity $f_{i H}$ defined there is equal to 1 for every $i \in E$. Then, $\left(y_{i}^{*}: i \in E\right)$ coincides with $\left(e_{i H}(\lambda)\right.$ : $i \in E$ ) used in [4 Lemma 4.48]. Moreover, by the proof given on [4 page 148], we have $\mathbb{E}_{i} \exp \left(\lambda \sigma_{H}\right)=1+\lambda y_{i}^{*}$ for every $i \in E$. Besides, it can be 
checked that $\left(1+\lambda y_{i}^{*}: i \in E\right)$ is a nonnegative solution to equation (7.1). Hence $\mathbb{E}_{i} \exp \left(\lambda \sigma_{H}\right)=1+\lambda y_{i}^{*} \geqslant x_{i}^{*}$ for every $i \in E$, where $\left(x_{i}^{*}: i \in E\right)$ is the minimal nonnegative solution to equation (7.1). We are now going to prove that $\mathbb{E}_{i} \exp \left(\lambda \sigma_{H}\right)=x_{i}^{*}$ for all $i \in E$. The proof is split into two parts: either $\lambda \geqslant 0$ or $\lambda<0$.

First, let $\lambda \geqslant 0$. It is easily seen that $\left(x_{i}^{*}-1: i \in E\right)$ is a nonnegative solution to the equation

$$
y_{i}=\frac{1}{q_{i}-\lambda} \sum_{k \notin H \cup\{i\}} q_{i k} y_{k}+\frac{\lambda}{q_{i}-\lambda}, \quad i \in E .
$$

Hence, $x_{i}^{*}-1 \geqslant \lambda y_{i}^{*}$ since $\left(\lambda y_{i}^{*}\right)$ is the minimal nonnegative solution to the equation above, by the linear combination theorem [4: Theorem $2.12(1)]$. That is, $x_{i}^{*} \geqslant 1+\lambda y_{i}^{*}$. Combining what we have proved in the last paragraph, it follows that $x_{i}^{*}=\mathbb{E}_{i} \exp \left(\lambda \sigma_{H}\right)$ for all $i \in E$.

Next, let $\lambda<0$. Denote by $\left(\bar{y}_{i}: i \in E\right)$ the minimal nonnegative solution to the equation

$$
y_{i}=\frac{1}{q_{i}-\lambda} \sum_{k \notin H \cup\{i\}} q_{i k} y_{k}+\left[1-\frac{1}{q_{i}-\lambda} \sum_{k \notin H \cup\{i\}} q_{i k}\right], \quad i \in E .
$$

Clearly, we have $\bar{y}_{i} \leqslant 1$ since $y_{i} \equiv 1$ is a solution to the equation. We claim that $\bar{y}_{i} \equiv 1$. To see this, note that $\left(1-\bar{y}_{i}: i \in E\right)$ is the maximal solution to the equation

$$
y_{i}=\frac{1}{q_{i}-\lambda} \sum_{k \notin H \cup\{i\}} q_{i k} y_{k}, \quad 0 \leqslant y_{i} \leqslant 1, \quad i \in E .
$$

By a comparison lemma [4: Lemma 3.14], it suffices to show that the equation

$$
y_{i}=\frac{1}{q_{i}} \sum_{k \notin H \cup\{i\}} q_{i k} y_{k}, \quad 0 \leqslant y_{i} \leqslant 1, \quad i \in E
$$

has only trivial (i.e. zero-) solution. Then this follows by the recurrence assumption and 4. Lemma 4.46]. We remark that there is an alternative way to prove that $\bar{y}_{i} \equiv 1$, using the uniqueness rather than the recurrence assumption. Actually, equation (7.3) is an exit equation for a modified $Q$-matrix (any local modification of a $Q$-matrix does not interfere the uniqueness). The exit solution to (7.3) should be zero by uniqueness assumption.

We now return to our main proof. By the linear combination theorem [4, Theorem $2.12(1)],\left(x_{i}^{*}-\lambda y_{i}^{*}: i \in E\right)$ is the minimal nonnegative solution to equation (7.2). Hence $x_{i}^{*}-\lambda y_{i}^{*}=\bar{y}_{i} \equiv 1$ as we have just proved in the last paragraph. Therefore we conclude that $x_{i}^{*}=1+\lambda y_{i}^{*}=\mathbb{E}_{i} \exp \left(\lambda \sigma_{H}\right)$ for all $i \in E$. We have thus completed the proof of the lemma.

Now we present our results about the exponential moments of the return time $\sigma_{0}$, which can be referred in [18]. 
Proposition 7.2 Let the single birth $Q$-matrix $\left(q_{i j}\right)$ be irreducible. Assume that its process is ergodic. Define $\left(\widetilde{F}_{k}^{(i)}\right)$ and $\left(\tilde{d}_{k}\right)$ by setting $c_{i} \equiv \lambda>0$. Then for small $\lambda$,

$$
\mathbb{E}_{0} e^{\lambda \sigma_{0}}=\frac{q_{01}(1+\lambda \tilde{d})}{q_{01}-\lambda}<\infty \quad \text { and } \mathbb{E}_{n} e^{\lambda \sigma_{0}}=1+\lambda \sum_{k=0}^{n-1}\left(\widetilde{F}_{k}^{(0)} \tilde{d}-\tilde{d}_{k}\right)<\infty, \quad n \geqslant 1
$$

iff

$$
\tilde{d}:=\varlimsup_{n \rightarrow \infty} \mathbb{1}_{\left\{\sum_{k=0}^{n} \widetilde{F}_{k}^{(0)}>0\right\}} \frac{\sum_{k=0}^{n} \tilde{d}_{k}}{\sum_{k=0}^{n} \widetilde{F}_{k}^{(0)}}<\infty
$$

and

$$
\tilde{d} \sum_{k=0}^{n-1} \widetilde{F}_{k}^{(0)}>\sum_{k=0}^{n-1} \tilde{d}_{k} \quad \text { whenever } \sum_{k=0}^{n-1} \widetilde{F}_{k}^{(0)} \leqslant 0 \quad \text { for } n \geqslant 2 .
$$

Furthermore, once $\widetilde{F}_{n}^{(0)}>0$ for large enough $n$ and $\sum_{n} \widetilde{F}_{n}^{(0)}=\infty$, we have

$$
\tilde{d}=\lim _{n \rightarrow \infty} \frac{\tilde{d}_{n}}{\widetilde{F}_{n}^{(0)}} \text { if the limit exists. }
$$

Finally, the process is exponentially ergodic iff both $\tilde{d}<\infty$ and (7.4) holds.

Proof Let $\lambda \in\left(0, q_{i}\right)$ for every $i \in E$ and set $H=\{0\}$. Then by Lemma 7.1, $\left(\mathbb{E}_{i} \mathrm{e}^{\lambda \sigma_{0}}: i \in E\right)$ is the minimal solution $\left(x_{i}^{*}\right)$ of the following equation

$$
x_{i}=\frac{1}{q_{i}-\lambda} \sum_{k \notin\{0, i\}} q_{i k} x_{k}+\frac{q_{i 0}\left(1-\delta_{i 0}\right)}{q_{i}-\lambda}, \quad x_{i} \geqslant 1, \quad i \in E .
$$

Assume that $x_{i}^{*}<\infty$ for every $i \in E$ for a moment, and let $\left(x_{i}\right)$ be a finite nonnegative solution to the last equation. Then, by (4.2), we have

$$
(Q x)_{i}+\lambda x_{i}=q_{i 0}\left(x_{0}-1\right), \quad i \geqslant 1 ; \quad(Q x)_{0}+\lambda x_{0}=0 .
$$

Applying Theorem 1.1 to $c_{i} \equiv \lambda$ and $f_{i}=q_{i 0}\left(1-\delta_{i 0}\right)\left(x_{0}-1\right)$ for all $i \geqslant 0$, we obtain

$$
\begin{aligned}
x_{n} & =x_{0}\left(1-\lambda \sum_{k=0}^{n-1} \sum_{j=0}^{k} \frac{\widetilde{F}_{k}^{(j)}}{q_{j, j+1}}\right)+\left(x_{0}-1\right) \sum_{k=1}^{n-1} \sum_{j=1}^{k} \frac{\widetilde{F}_{k}^{(j)} q_{j 0}}{q_{j, j+1}} \\
& =x_{0}\left(1-\lambda \sum_{k=0}^{n-1} \sum_{j=0}^{k} \frac{\widetilde{F}_{k}^{(j)}}{q_{j, j+1}}\right)+\left(x_{0}-1\right) \sum_{k=1}^{n-1} \sum_{j=1}^{k} \frac{\widetilde{F}_{k}^{(j)}\left(\tilde{q}_{j}^{(0)}+\lambda\right)}{q_{j, j+1}}, \quad n \geqslant 1 .
\end{aligned}
$$

Due to the explicit representation of $\widetilde{F}_{n}^{(k)}, \widetilde{m}_{n}$ and $\tilde{d}_{n}$, given in (2.7), (3.2) and (5.2) respectively, we have not only

$$
\tilde{m}_{n}=\sum_{0 \leqslant j \leqslant n} \frac{\widetilde{F}_{n}^{(j)}}{q_{j, j+1}}=\frac{1}{q_{01}} \widetilde{F}_{n}^{(0)}+\tilde{d}_{n}, \quad n \geqslant 0
$$


but also that

$$
\begin{aligned}
x_{n} & =x_{0}\left(1-\lambda \sum_{k=0}^{n-1} \tilde{m}_{k}\right)+\left(x_{0}-1\right) \sum_{k=1}^{n-1}\left(\widetilde{F}_{k}^{(0)}+\lambda \tilde{d}_{k}\right) \\
& =x_{0}\left(1-\frac{\lambda}{q_{01}}\right) \sum_{k=0}^{n-1} \widetilde{F}_{k}^{(0)}-\sum_{k=0}^{n-1}\left(\widetilde{F}_{k}^{(0)}+\lambda \tilde{d}_{k}\right)+1, \quad n \geqslant 1 .
\end{aligned}
$$

Since $x_{n}>1$, we get

$$
x_{0}\left(1-\frac{\lambda}{q_{01}}\right) \sum_{k=0}^{n-1} \widetilde{F}_{k}^{(0)}>\sum_{k=0}^{n-1}\left(\widetilde{F}_{k}^{(0)}+\lambda \tilde{d}_{k}\right), \quad n \geqslant 1 .
$$

That is

$$
\left[x_{0}\left(\frac{1}{\lambda}-\frac{1}{q_{01}}\right)-\frac{1}{\lambda}\right] \sum_{k=0}^{n-1} \widetilde{F}_{k}^{(0)}>\sum_{k=0}^{n-1} \tilde{d}_{k}, \quad n \geqslant 1 .
$$

Note that on the one hand, if $x_{0}^{*}=x_{0}^{*}\left(\lambda_{0}\right)<\infty$, then $x_{0}^{*}=x_{0}^{*}(\lambda)<\infty$ for every $\lambda \in\left(0, \lambda_{0}\right)$, by the comparison theorem (cf. [4 Theorem 2.6]). On the other hand, when $\lambda=0$, we have

$$
\sum_{k=0}^{n} \widetilde{F}_{k}^{(0)}=\sum_{k=0}^{n} F_{k}^{(0)}>0 \quad \text { and } \quad \sum_{k=0}^{n} \tilde{d}_{k}=\sum_{k=0}^{n} d_{k}>0, \quad n \geqslant 1 .
$$

For each fixed $n, \sum_{k=0}^{n} \widetilde{F}_{k}^{(0)}$ and $\sum_{k=0}^{n} \tilde{d}_{k}$ are analytic in $\lambda$, and so should be positive for sufficient small $\lambda$, say $\lambda \leqslant \lambda_{1}$ for some $\lambda_{1} \leqslant \lambda_{0}$. Then by (7.8), we should have

$$
x_{0}\left(\frac{1}{\lambda}-\frac{1}{q_{01}}\right)-\frac{1}{\lambda}>0, \quad \lambda \in\left(0, \lambda_{1}\right)
$$

independent of $n$. Therefore, by the minimal property, we have

$$
x_{0}^{*}\left(\frac{1}{\lambda}-\frac{1}{q_{01}}\right)-\frac{1}{\lambda}=\varlimsup_{n \rightarrow \infty} \mathbb{1}_{\left\{\sum_{k=0}^{n} \widetilde{F}_{k}^{(0)}>0\right\}}\left[\sum_{k=0}^{n} \tilde{d}_{k}\right]\left[\sum_{k=0}^{n} \widetilde{F}_{k}^{(0)}\right]^{-1}=\tilde{d},
$$

i.e.

$$
\mathbb{E}_{0} \mathrm{e}^{\lambda \sigma_{0}}=x_{0}^{*}=\frac{q_{01}(1+\lambda \tilde{d})}{q_{01}-\lambda} .
$$

Since $x_{0}^{*}$ satisfies (7.8), we obtain condition (7.4). Then

$$
\mathbb{E}_{n} \mathrm{e}^{\lambda \sigma_{0}}=1+\lambda \sum_{k=0}^{n-1}\left(\widetilde{F}_{k}^{(0)} \tilde{d}-\tilde{d}_{k}\right), \quad n \geqslant 1 .
$$

Conversely, if $\tilde{d}<\infty$ and (7.4) holds. Then starting from $x_{0}=x_{0}^{*}$ given in (7.9) and defining $x_{n}$ by (7.7), we obtain a solution $\left(x_{i}>1: i \in E\right)$ to (7.5). 
By (4.2), we obtain a finite nonnegative solution to the original equation for $\left(\mathbb{E}_{i} e^{\lambda \sigma_{0}}: i \in E\right)$, and hence the minimal solution $\left(x_{i}^{*}=\mathbb{E}_{i} e^{\lambda \sigma_{0}}: i \in E\right)$ should be finite.

Finally, by [4: Theorem 4.44], the process is exponentially ergodic iff $\mathbb{E}_{0} \mathrm{e}^{\lambda \sigma_{0}}$ $<\infty$, equivalently, $\tilde{d}<\infty$ and (7.4) holds. The last assertion of the proposition then follows.

In contract to the ergodic case, one may study the exponential decay (in the transient case) for which the Poisson equation becomes

$$
Q g+\lambda g=0, \quad g>0 .
$$

With $c_{i} \equiv \lambda$, by Theorem 1.1, the solution is

$$
g_{n}=g_{0}\left[1-\lambda \sum_{0 \leqslant k \leqslant n-1} \sum_{0 \leqslant j \leqslant k} \frac{\widetilde{F}_{k}^{(j)}}{q_{j, j+1}}\right]=g_{0}\left[1-\lambda \sum_{0 \leqslant k \leqslant n-1} \tilde{m}_{k}\right], \quad n \geqslant 0 .
$$

This is somehow simpler than the previous one. However, these two exponential cases are actually much harder than the others, for instance we do not know at the moment how to remove condition (7.4). That is showing for some $\lambda>0$, small enough, $\sum_{k=0}^{n} \widetilde{F}_{k}^{(0)}>0$ for all $n$ (or equivalently, $\underline{\lim }_{n \rightarrow \infty} \sum_{k=0}^{n} \widetilde{F}_{k}^{(0)}>0$ ). This seems necessary for the exponential ergodicity since $\sum_{k=0}^{\infty} \widetilde{F}_{k}^{(0)}=\infty$ when $\lambda=0$ by the recurrence (which is much weaker than exponential ergodicity) and $\lambda$ is allowed to be very small. Actually, to figure out a criterion, one needs much more work using different approaches, refer to [4: Chapter 9] and [7] for some details.

\section{Laplace transform of the return/extinction time}

Note that for negative $\lambda, \mathbb{E}_{i} \mathrm{e}^{\lambda \sigma_{0}}$ is the Laplace transform of $\sigma_{0}$. The proof of Proposition 7.2 is still available. So we get the following result.

Proposition 7.3 Define $\left(\widetilde{F}_{k}^{(i)}\right)$ and $\left(\tilde{d}_{k}\right)$ by (1.1) and (5.1), respectively, with $c_{i} \equiv-\lambda<0$. Let the single birth process be recurrent. Then the Laplace transform of $\sigma_{0}$ is given by

$$
\mathbb{E}_{0} e^{-\lambda \sigma_{0}}=\frac{q_{01}(1-\lambda \tilde{d})}{q_{01}+\lambda}, \quad \mathbb{E}_{n} e^{-\lambda \sigma_{0}}=1-\lambda \sum_{k=0}^{n-1}\left(\widetilde{F}_{k}^{(0)} \tilde{d}-\tilde{d}_{k}\right), \quad n \geqslant 1,
$$

where

$$
\tilde{d}=\lim _{n \rightarrow \infty} \frac{\sum_{k=0}^{n-1} \tilde{d}_{k}}{\sum_{k=0}^{n-1} \widetilde{F}_{k}^{(0)}}=\lim _{n \rightarrow \infty} \frac{\tilde{d}_{n}}{\widetilde{F}_{n}^{(0)}} \quad \text { if the limit exists. }
$$


Proof Following the proof of Proposition 7.2 , replacing $\lambda$ by $-\lambda$, we arrive at

$$
\begin{aligned}
x_{n} & =x_{0}\left(1+\frac{\lambda}{q_{01}}\right) \sum_{k=0}^{n-1} \widetilde{F}_{k}^{(0)}-\sum_{k=0}^{n-1}\left(\widetilde{F}_{k}^{(0)}-\lambda \tilde{d}_{k}\right)+1, \\
& =: x_{0} \alpha_{n-1}-\beta_{n-1}, \quad n \geqslant 1 .
\end{aligned}
$$

By the minimal nonnegative property, $x_{0}^{*}=\sup _{n \geqslant 1} \beta_{n} / \alpha_{n}$, and then we indeed have

$$
x_{0}^{*}=\varlimsup_{n \rightarrow \infty} \frac{\beta_{n}}{\alpha_{n}} .
$$

We now show that we can replace $\varlimsup_{n \rightarrow \infty}$ by $\lim _{n \rightarrow \infty}$. Noting that on the one hand, since $x_{n} \in(0,1]$, we have

$$
\frac{\beta_{n}}{\alpha_{n}}<x_{0} \leqslant \frac{\beta_{n}+1}{\alpha_{n}}, \quad n \geqslant 1 .
$$

On the other hand, following the proof for

$$
\sum_{k} \tilde{m}_{k}=\infty \Longleftrightarrow \sum_{k} m_{k}=\infty
$$

given in Section 3, we can prove that $\sum_{k} \widetilde{F}_{k}^{(0)}=\infty$ since $\sum_{k} F_{k}^{(0)}=\infty$ by the recurrent assumption (i.e. $\gamma_{j} \equiv 1$ ). Hence we can rewrite $\varlimsup_{n \rightarrow \infty} \beta_{n} / \alpha_{n}$ as $\lim _{n \rightarrow \infty} \beta_{n} / \alpha_{n}$. Therefore, we have

$$
\begin{aligned}
x_{0}^{*} & =\lim _{n \rightarrow \infty}\left[\sum_{k=0}^{n-1}\left(\widetilde{F}_{k}^{(0)}-\lambda \tilde{d}_{k}\right)\right]\left\{\left[1+\frac{\lambda}{q_{01}}\right] \sum_{k=0}^{n-1} \widetilde{F}_{k}^{(0)}\right\}^{-1} \\
& =\frac{q_{01}}{q_{01}+\lambda} \lim _{n \rightarrow \infty}\left[1-\lambda \frac{\sum_{k=0}^{n-1} \tilde{d}_{k}}{\sum_{k=0}^{n-1} \widetilde{F}_{k}^{(0)}}\right] \\
& =\frac{q_{01}}{q_{01}+\lambda}[1-\lambda \tilde{d}] .
\end{aligned}
$$

Furthermore,

$x_{n}^{*}=(1-\lambda \tilde{d}) \sum_{k=0}^{n-1} \widetilde{F}_{k}^{(0)}-\sum_{k=0}^{n-1}\left(\widetilde{F}_{k}^{(0)}-\lambda \tilde{d}_{k}\right)+1=1-\lambda \sum_{k=0}^{n-1}\left(\widetilde{F}_{k}^{(0)} \tilde{d}-\tilde{d}_{k}\right), \quad n \geqslant 1$.

The last limit in $\tilde{d}$ is an application of Stolz's Theorem.

\section{Exponential moments and Laplace transform of the life time}

Now we return to $\tau_{\infty}$. 
Proposition 7.4 Assume that the single birth $Q$-matrix $Q=\left(q_{i j}\right)$ is explosive and irreducible. Define $\left(\widetilde{m}_{k}\right)$ by (3.1) with $c_{i} \equiv \lambda$. For the corresponding minimal process,

(i) if there exists a $\lambda>0$ such that $\lambda \sum_{k=0}^{n-1} \tilde{m}_{k}<1$ for every $n>1$, then

$$
\mathbb{E}_{n} e^{\lambda \tau_{\infty}}=1+\lambda\left[\bar{c}\left(1-\lambda \sum_{k=0}^{n-1} \tilde{m}_{k}\right)-\sum_{k=0}^{n-1} \tilde{m}_{k}\right], \quad n \geqslant 0,
$$

where

$$
\bar{c}=\varlimsup_{n \rightarrow \infty} \frac{\sum_{k=0}^{n} \tilde{m}_{k}}{1-\lambda \sum_{k=0}^{n} \tilde{m}_{k}} .
$$

Furthermore, the process decays exponentially fast provided $\bar{c}<\infty$.

(ii) For $\lambda>0$, the Laplace transform of $\tau_{\infty}$ is given by

$$
\mathbb{E}_{n} e^{-\lambda \tau_{\infty}}=\frac{1+\lambda \sum_{0 \leqslant k \leqslant n-1} \tilde{m}_{k}}{1+\lambda \sum_{k \geqslant 0} \tilde{m}_{k}}, \quad n \geqslant 0 .
$$

Proof Define

$$
e_{i \infty}(\lambda)=\int_{0}^{\infty} \mathrm{e}^{\lambda t} \mathbb{P}_{i}\left(\tau_{\infty}>t\right) \mathrm{d} t
$$

with $\lambda<q_{i}$ for all $i \geqslant 0$. Note that the process is explosive and

$$
\mathbb{E}_{i} \mathrm{e}^{\lambda \tau_{\infty}}=1+\lambda e_{i \infty}(\lambda) .
$$

Because $\mathbb{P}_{m}\left(\tau_{n}<\eta\right)=1$ for every pair $m<n$, we have $\mathbb{P}_{m}\left(\tau_{n}<\infty\right)=1$ and furthermore $\mathbb{P}_{m}\left(\tau_{\infty}<\infty\right)=1$ for every $m$, as $n$ goes to $\infty$. Then by [4. Lemma 4.48], $\left(e_{i \infty}(\lambda)\right)$ is the minimal solution to the equation

$$
x_{i}=\frac{q_{i}}{q_{i}-\lambda} \sum_{k} \Pi_{i k} x_{k}+\frac{1}{q_{i}-\lambda}, \quad i \geqslant 0 .
$$

By (4.2), we can rewrite the equation as

$$
(Q x)_{i}+\lambda x_{i}=-1, \quad i \geqslant 0 .
$$

Applying Theorem 1.1 to $c_{i} \equiv \lambda$ and $f_{i} \equiv-1$, the solution of the equation has the form:

$$
\begin{aligned}
x_{n} & =x_{0}\left(1-\lambda \sum_{k=0}^{n-1} \sum_{j=0}^{k} \frac{\widetilde{F}_{k}^{(j)}}{q_{j, j+1}}\right)-\sum_{k=0}^{n-1} \sum_{j=0}^{k} \frac{\widetilde{F}_{k}^{(j)}}{q_{j, j+1}} \\
& =x_{0}\left(1-\lambda \sum_{k=0}^{n-1} \tilde{m}_{k}\right)-\sum_{k=0}^{n-1} \tilde{m}_{k}, \quad n \geqslant 1 .
\end{aligned}
$$

Note that $\lambda<q_{0}=q_{01}$ and $\lambda \widetilde{m}_{0}<1$. If there exists a positive $\lambda$ small enough so that $\lambda \sum_{k=0}^{n-1} \widetilde{m}_{k}<1$ for every $n>1$, then by the argument above and the minimal property of the solution, one gets

$$
e_{0 \infty}(\lambda)=\sup _{n \geqslant 1} \frac{\sum_{k=0}^{n-1} \tilde{m}_{k}}{1-\lambda \sum_{k=0}^{n-1} \tilde{m}_{k}}=\varlimsup_{n \rightarrow \infty} \frac{\sum_{k=0}^{n} \tilde{m}_{k}}{1-\lambda \sum_{k=0}^{n} \tilde{m}_{k}}=: \bar{c}
$$


and

$$
e_{n \infty}(\lambda)=\bar{c}\left(1-\lambda \sum_{k=0}^{n-1} \widetilde{m}_{k}\right)-\sum_{k=0}^{n-1} \widetilde{m}_{k}, \quad n \geqslant 1 .
$$

Then the first assertion follows.

For the Laplace transform of $\tau_{\infty}$, the argument above still works because now we deal with the case of $-\lambda<0$. By the explosive property, we know that $\sum_{k=0}^{\infty} \tilde{m}_{k}<\infty$. Hence we have

$$
e_{0 \infty}(-\lambda)=\bar{c}=\frac{\sum_{k=0}^{\infty} \tilde{m}_{k}}{1+\lambda \sum_{k=0}^{\infty} \tilde{m}_{k}}
$$

and

$$
e_{n \infty}(-\lambda)=\bar{c}\left(1+\lambda \sum_{k=0}^{n-1} \tilde{m}_{k}\right)-\sum_{k=0}^{n-1} \tilde{m}_{k}=\frac{\sum_{k=n}^{\infty} \tilde{m}_{k}}{1+\lambda \sum_{k=0}^{\infty} \tilde{m}_{k}}, \quad n \geqslant 1 .
$$

Finally, we have

$$
\mathbb{E}_{n} \mathrm{e}^{-\lambda \tau_{\infty}}=1-\frac{\lambda \sum_{k=n}^{\infty} \tilde{m}_{k}}{1+\lambda \sum_{k=0}^{\infty} \tilde{m}_{k}}=\frac{1+\lambda \sum_{0 \leqslant k \leqslant n-1} \tilde{m}_{k}}{1+\lambda \sum_{k \geqslant 0} \tilde{m}_{k}}, \quad n \geqslant 0 .
$$

The proof for the second assertion is now finished.

A more careful study on part (i) of Proposition 7.4, refer to Proposition 7.2,

\section{Examples}

In the special case of birth-death processes, the problems studied here have rather complete solutions, see for instance [4. Theorem 4.55]. As mentioned in the introduction of the paper, much more models have been studied in the past years. Here we make a little addition. The next example is taken from [3].

Example 8.1 (uniform catastrophes) Let

$$
q_{i, i+1}=b i, \quad i \geqslant 0 ; \quad q_{i j}=a, \quad j=0,1, \ldots, i-1 ;
$$

and $q_{i j}=0$ for other $j>i+1$, where $a$ and $b$ are positive constants. Then the extinction of the process has an exponential distribution

$$
\mathbb{E}_{n} \mathrm{e}^{-\lambda \tau_{0}}=\frac{a}{a+\lambda}, \quad \lambda>0, n \geqslant 1 .
$$

It is surprising that the distribution is independent of $b$ and the starting point $n$. Redefine $q_{01}=1$. Then the irreducible process is indeed strongly ergodic. 
Proof We need to consider the case that $q_{01}>0$ only. With $c_{i} \equiv-\lambda \in \mathbb{R}$ and then $\tilde{q}_{n}^{(k)}=(k+1) a+\lambda$ for $k \leqslant n-1$, by using (1.1), (5.1), and induction, one may check that

$$
\begin{gathered}
\tilde{F}_{n}^{(0)}=\frac{a+\lambda}{n b} \prod_{1 \leqslant k \leqslant n-1}\left(1+\frac{(k+1) a+\lambda}{k b}\right), \quad \prod_{\varnothing}=: 1, \\
\tilde{d}_{n}=\frac{1}{n b} \prod_{1 \leqslant k \leqslant n-1}\left(1+\frac{(k+1) a+\lambda}{k b}\right), \quad n \geqslant 1 .
\end{gathered}
$$

Since for each fixed $\lambda \in \mathbb{R}$,

$$
\log \left(1+\frac{(n+1) a+\lambda}{n b}\right) \rightarrow \log \left(1+\frac{a}{b}\right)>0 \quad \text { as } n \rightarrow \infty,
$$

we have $\lim _{n \rightarrow \infty} \widetilde{F}_{n}^{(0)}=\infty$ and so $\sum_{n} \widetilde{F}_{n}^{(0)}=\infty$. As an application of this fact with $\lambda=0$, it follows that the process is recurrent (Proposition 4.1) and then should be non-explosive ( $(7.6)$ and Proposition 3.1).

Next, because

$$
\sum_{n} \widetilde{F}_{n}^{(0)}=\infty, \quad \widetilde{F}_{n}^{(0)}=(a+\lambda) \tilde{d}_{n}, \quad n \geqslant 1,
$$

it follows that

$$
\tilde{d}=\lim _{n \rightarrow \infty} \frac{\tilde{d}_{n}}{\widetilde{F}_{n}^{(0)}}=\frac{1}{a+\lambda}
$$

Hence, we have

$$
\widetilde{F}_{n}^{(0)} \tilde{d}=\tilde{d}_{n}, \quad n \geqslant 1,
$$

From here, when $\lambda=0$ in particular, we obtain

$$
\sup _{k} \sum_{n=0}^{k}\left(F_{n}^{(0)} d-d_{n}\right)=d=a^{-1}<\infty .
$$

Hence the process is strongly ergodic by Proposition 5.2 .

By using Proposition 7.3, we obtain

$$
\begin{aligned}
& \mathbb{E}_{0} \mathrm{e}^{-\lambda \sigma_{0}}=\frac{a q_{01}}{(a+\lambda)\left(q_{01}+\lambda\right)}, \\
& \mathbb{E}_{n} \mathrm{e}^{-\lambda \sigma_{0}}=1-\lambda \tilde{d}=\frac{a}{a+\lambda}=\mathbb{E}_{n} \mathrm{e}^{-\lambda \tau_{0}}, \quad n \geqslant 1 .
\end{aligned}
$$

Therefore, we have proved the first assertion. 
Even though it is now automatic that the process is exponentially ergodic, implied by the strongly ergodicity, we would like to check the effectiveness of Proposition 7.2 for this model. To do so, reset $c_{i} \equiv \lambda>0$. Then

$$
\begin{aligned}
\widetilde{F}_{n}^{(0)} & =\frac{a-\lambda}{n b} \prod_{1 \leqslant k \leqslant n-1}\left(1+\frac{(k+1) a-\lambda}{k b}\right), \\
\tilde{d}_{n} & =\frac{1}{n b} \prod_{1 \leqslant k \leqslant n-1}\left(1+\frac{(k+1) a-\lambda}{k b}\right), \quad n \geqslant 1 .
\end{aligned}
$$

Clearly, $\widetilde{F}_{n}^{(0)}>0$ and so does $\tilde{d}_{n}$ for every $\lambda \in(0, a)$. As we have proved above

$$
\sum_{n} \widetilde{F}_{n}^{(0)}=\infty, \quad \tilde{d}=\lim _{n \rightarrow \infty} \frac{\tilde{d}_{n}}{\widetilde{F}_{n}^{(0)}}=\frac{1}{a-\lambda}<\infty,
$$

and hence the process is exponentially ergodic by Proposition 7.2. Actually, we have

$$
\begin{aligned}
\mathbb{E}_{0} \mathrm{e}^{\lambda \sigma_{0}} & =\frac{a q_{01}}{(a-\lambda)\left(q_{01}-\lambda\right)}, \\
\mathbb{E}_{n} \mathrm{e}^{\lambda \sigma_{0}} & =\frac{a}{a-\lambda}, \quad n \geqslant 1, \quad \lambda \in\left(0, a \wedge q_{01}\right) .
\end{aligned}
$$

Example 8.2 Consider the single birth $Q$-matrix $\left(q_{i j}\right)$ with

$$
q_{i 0}>0, \quad q_{i, i+1}>0, q_{i j}=0 \text { for all other } j \neq i .
$$

Let $c_{i} \in \mathbb{R}$. Then

(1) we have

$$
\begin{gathered}
\widetilde{F}_{i}^{(i)}=1, \quad \widetilde{F}_{n}^{(i)}=\frac{q_{n 0}-c_{n}}{q_{n, n+1}} \prod_{i+1 \leqslant k \leqslant n-1}\left[1+\frac{q_{k 0}-c_{k}}{q_{k, k+1}}\right], \\
\prod_{\varnothing}=: 1, \quad n>i \geqslant 0,
\end{gathered}
$$

and then $\left(\tilde{m}_{n}\right)$ and $\left(\tilde{d}_{n}\right)$ are given by (3.2) and (5.2), respectively.

(2) In particular, if $q_{n 0}-c_{n} \equiv q_{10}-c_{1}$ for every $n \geqslant 1$, then

$$
\begin{gathered}
\widetilde{F}_{i}^{(i)}=1, \quad \widetilde{F}_{n}^{(i)}=\frac{q_{10}-c_{1}}{q_{n, n+1}} \prod_{k=i+1}^{n-1}\left[1+\frac{q_{10}-c_{1}}{q_{k, k+1}}\right], \prod_{\varnothing}=: 1, \quad n>i \geqslant 0, \\
\tilde{m}_{0}=\frac{1}{q_{01}}, \quad \tilde{m}_{n}=\frac{1}{q_{n, n+1}} \prod_{k=0}^{n-1}\left[1+\frac{q_{10}-c_{1}}{q_{k, k+1}}\right], \quad n \geqslant 1, \\
\tilde{d}_{0}=0, \quad \tilde{d}_{n}=\frac{1}{q_{n, n+1}} \prod_{1 \leqslant k \leqslant n-1}\left[1+\frac{q_{10}-c_{1}}{q_{k, k+1}}\right], \quad n \geqslant 1 .
\end{gathered}
$$


Furthermore, the process is explosive if

$$
\kappa^{\prime}:=\lim _{n \rightarrow \infty} \frac{n\left(q_{n+1, n+2}-q_{n, n+1}-q_{10}\right)}{q_{n, n+1}+q_{10}}>1
$$

$\left(q_{n, n+1}=(n+1)^{\gamma}\right.$ for $\gamma>1$ for example). Otherwise, if $\kappa^{\prime}<1\left(q_{n, n+1}=\right.$ $(n+1)^{\gamma}$ for some $\gamma \leqslant 1$ for instance), then the process is unique. If so, the process is indeed strongly ergodic.

Proof (a) By assumption, we have $\tilde{q}_{n}^{(k)}=q_{n 0}-c_{n}$ for every $k<n$. Hence, by (1.1), we obtain

$$
\widetilde{F}_{n}^{(i)}=\frac{\tilde{q}_{n}^{(0)}}{q_{n, n+1}} \sum_{k=i}^{n-1} \widetilde{F}_{k}^{(i)} .
$$

Thus, to prove (8.1), it suffices to show that

$$
\sum_{k=i}^{n-1} \widetilde{F}_{k}^{(i)}=\prod_{i+1 \leqslant k \leqslant n-1}\left[1+\frac{\tilde{q}_{k}^{(0)}}{q_{k, k+1}}\right], \quad n>i \geqslant 0 .
$$

This clearly holds when $n=i+1$. Suppose that it holds when $n=\ell$, then

$$
\begin{aligned}
\sum_{k=i}^{\ell} \widetilde{F}_{k}^{(i)} & =\sum_{k=i}^{\ell-1} \widetilde{F}_{k}^{(i)}+\widetilde{F}_{\ell}^{(i)} \\
& =\sum_{k=i}^{\ell-1} \widetilde{F}_{k}^{(i)}+\frac{\tilde{q}_{\ell}^{(0)}}{q_{\ell, \ell+1}} \sum_{k=i}^{\ell-1} \widetilde{F}_{k}^{(i)} \quad(\text { by }(\underline{8.2})) \\
& =\left[1+\frac{\tilde{q}_{\ell}^{(0)}}{q_{\ell, \ell+1}}\right] \sum_{k=i}^{\ell-1} \widetilde{F}_{k}^{(i)} \\
& =\prod_{i+1 \leqslant k \leqslant \ell}\left[1+\frac{\tilde{q}_{\ell}^{(0)}}{q_{k, k+1}}\right] \quad \text { (by inductive assumption). }
\end{aligned}
$$

Therefore, the required assertion holds for $n=\ell$ and it then holds for all $n>i$ by induction. We have thus proved the first assertion.

(b) By assumption, we have $\tilde{q}_{n}^{(k)}=q_{10}-c_{1}$ for every $k<n$. Hence, by (3.1) and (5.1), we obtain

$$
\begin{array}{cl}
\tilde{m}_{n}=\frac{1}{q_{n, n+1}}\left(1+\tilde{q}_{1}^{(0)} \sum_{k=0}^{n-1} \tilde{m}_{k}\right), & n \geqslant 1, \\
\tilde{d}_{n}=\frac{1}{q_{n, n+1}}\left(1+\tilde{q}_{1}^{(0)} \sum_{k=0}^{n-1} \tilde{d}_{k}\right), & n \geqslant 1 .
\end{array}
$$

As in the last proof, by using induction, we obtain the explicit expressions of $\left(\tilde{m}_{n}\right)$ and $\left(\tilde{d}_{n}\right)$. 
To study the divergence of $\sum_{n} m_{n}$, we adopt the

Kummer Test Let $\left(u_{n}\right)$ and $\left(v_{n}\right)$ be two sequences of positive numbers. Suppose that $\sum_{0}^{\infty} 1 / v_{n}=\infty$ and the limit $\kappa:=\lim _{n \rightarrow \infty} \kappa_{n}$ exists, where

$$
\kappa_{n}=v_{n} \cdot \frac{u_{n}}{u_{n+1}}-v_{n+1} \text {. }
$$

Then, the series $\sum u_{n}$ converges or diverges according to $\kappa>0$ or $\kappa<0$ respectively.

Set $v_{n} \equiv n$ and $u_{n}=m_{n}$ :

$$
m_{n}=\frac{1}{q_{n, n+1}} \prod_{0 \leqslant k \leqslant n-1}\left[1+\frac{q_{10}}{q_{k, k+1}}\right], \quad n \geqslant 0 .
$$

Then

$$
v_{n} \frac{u_{n}}{u_{n+1}}-v_{n+1}=\frac{n\left(q_{n+1, n+2}-q_{n, n+1}-q_{10}\right)}{q_{n, n+1}+q_{10}}-1 .
$$

Hence $\sum_{n} u_{n}<\infty$ if $\kappa^{\prime}>1$ (resp. $\sum_{n} u_{n}=\infty$ once $\left.\kappa^{\prime}<1\right)$. Clearly, $\sum_{n} m_{n}=$ $\infty$ implies $\sum_{n} F_{n}^{(0)}=\infty$. Hence

$$
d=\lim _{n \rightarrow \infty} \frac{d_{n}}{F_{n}^{(0)}}=\frac{1}{q_{01}} .
$$

Furthermore,

$$
\sup _{k \in E} \sum_{n=0}^{k}\left(F_{n}^{(0)} d-d_{n}\right)=F_{0}^{(0)} d=d<\infty .
$$

This gives us the strong ergodicity by Proposition 5.2 .

We mention that Proposition 7.2 (with $0<c_{i} \equiv \lambda<q_{10}$ ) is also available for this model.

Remark 8.3 For exponential ergodicity, the following sufficient condition

$$
M:=\sup _{n \geqslant 1}\left[\sum_{k=1}^{n-1} F_{k}^{(0)}\right]\left[\sum_{j=n}^{\infty} \frac{1}{q_{j, j+1} F_{j}^{(0)}}\right]<\infty,
$$

introduced in [12], is sufficient for Example 8.1 but is not for Example 8.2.

Proof It is obvious that $M<\infty$ iff

$$
\varlimsup_{n \rightarrow \infty}\left[\sum_{k=1}^{n-1} F_{k}^{(0)}\right]\left[\sum_{j=n}^{\infty} \frac{1}{q_{j, j+1} F_{j}^{(0)}}\right]<\infty .
$$

For Example 8.1, because $q_{j, j+1} F_{j}^{(0)}$ is growing exponentially fast and so it is easy to check that $M<\infty$. For Example 8.2, it suffices to consider $q_{n, n+1}=$ $b(n+1)$ for some $b>0$. By Kummer test, one may show that

$$
\sum_{j=n}^{\infty} \frac{1}{q_{j, j+1} F_{j}^{(0)}}=\infty
$$


for suitable $b>0$ and then $M=\infty$.

Acknowledgements The authors acknowledge the support by NNSFC (No. 11131003), SRFDP (No. 20100003110005), the "985" project from the Ministry of Education in China, the Fundamental Research Funds for the Central Universities, and the Project Funded by the Priority Academic Program Development of Jiangsu Higher Education Institutions.

\section{References}

1. Anderson W J. Continuous-Time Markov Chains: An Applications-Oriented Approach. New York: Springer-Verlag, 1991

2. Brockwell P J. The extinction time of a general birth and death processes with catastrophes. J Appl Prob, 1986, 23: 851-858

3. Brockwell P J, Gani J, Resnick S I. Birth, immigration and catastrophe processes. Adv Appl Prob, 1982, 14: 709-731

4. Chen M F. From Markov Chains to Non-Equilibrium Particle Systems (2nd Edition). Singapore: World Scientific, 2004

5. Chen M F. Single birth processes. Chinese Ann Math, 1999, 20B: 77-82

6. Chen M F. Explicit criteria for several types of ergodicity. Chinese J Appl Prob Stat, 2001, 17(2): 1-8

7. Chen M F. Speed of stability for birth-death process. Front Math China, 2010, 5(3): 379-516

8. Chen M F, Zhang X. Isospectral operators. 2014, preprint

9. Hou Z T, Guo Q F. Homogeneous Denumerable Markov Processes (in Chinese), Beijing: Science Press, 1978; English translation, Beijing: Science Press and Springer, 1988

10. Mao Y H. Ergodic degrees for continuous-time Markov chains. Science in China Ser A Mathematics, 2004, 47(2): 161-174

11. Mao Y H. Eigentime identity for transient Markov chains. J Math Anal Appl, 2006, 315(2): 415-424

12. Mao Y H, Zhang Y H. Exponential ergodicity for single-birth processes. J Applied Probab, 2004, 41: 1022-1032

13. Reuter G E H. Competition Processes. In Fourth Berkeley Symposium on Math Stat and Prob, 1961, 2: 421-430

14. Wang L D, Zhang Y H. Criteria for zero-exit (-entrance) of single-birth (-death) Qmatrices. Acta Math. Sinica, 2014, to appear (in Chinese)

15. Yan S J, Chen M F. Multidimensional Q-processes. Chinese Ann Math, 1986, 7B: 90-110

16. Zhang J K. On the generalized birth and death processes (I). Acta Math Sci, 1984, 4: $241-259$

17. Zhang Y H. Strong ergodicity for single-birth processes. J Appl Prob, 2001, 38(1): 270-277

18. Zhang Y H. Moments of the first hitting time for single birth processes. J Beijing Normal Univ, 2003, 39(4): 430-434 (in Chinese)

19. Zhang Y H. The hitting time and stationary distribution for single birth processes. J Beijing Normal Univ, 2004, 40(2): 157-161 (in Chinese)

20. Zhang Y H. Birth-death-catastrophe type single birth $Q$-matrices. J Beijing Normal Univ, 2011, 47(4): 347-350 (in Chinese)

21. Zhang Y H. Expressions on moments of hitting time for single birth process in infinite and finite space. J. Beijing Normal Univ, 2013, 49(5): 445-452 (in Chinese) 


\section{Appendix. Key formulas used in the proofs}

(A) Solution to the Poisson equation $\Omega g=Q g+c g$ :

$$
g_{n}=g_{0}+\sum_{0 \leqslant k \leqslant n-1} \sum_{0 \leqslant j \leqslant k} \frac{\widetilde{F}_{k}^{(j)}\left(f_{j}-c_{j} g_{0}\right)}{q_{j, j+1}}, \quad n \geqslant 0 .
$$

(B) Three sequences.

(a) $\widetilde{F}$-sequence:

$$
\widetilde{F}_{i}^{(i)}=1, \quad \widetilde{F}_{n}^{(i)}=\frac{1}{q_{n, n+1}} \sum_{k=i}^{n-1} \tilde{q}_{n}^{(k)} \widetilde{F}_{k}^{(i)}, \quad n>i \geqslant 0, \quad \text { (1.1) }
$$

where

$$
\tilde{q}_{n}^{(k)}=q_{n}^{(k)}-c_{n}:=\sum_{j=0}^{k} q_{n j}-c_{n}, \quad 0 \leqslant k<n .
$$

(b) $\tilde{m}$-sequence:

$$
\widetilde{m}_{0}=\frac{1}{q_{01}}, \quad \widetilde{m}_{n}=\frac{1}{q_{n, n+1}}\left(1+\sum_{k=0}^{n-1} \tilde{q}_{n}^{(k)} \tilde{m}_{k}\right), \quad n \geqslant 1 .
$$

(c) $\tilde{d}$-sequence:

$$
\tilde{d}_{0}=0, \quad \tilde{d}_{n}=\frac{1}{q_{n, n+1}}\left(1+\sum_{k=0}^{n-1} \tilde{q}_{n}^{(k)} \tilde{d}_{k}\right), \quad n \geqslant 1 .
$$

Representation of the three sequences:

$$
\begin{array}{lll}
\widetilde{F}_{i}^{(i)}=1, \quad \widetilde{F}_{n}^{(i)}=\sum_{k=i+1}^{n} \frac{\widetilde{F}_{n}^{(k)} \tilde{q}_{k}^{(i)}}{q_{k, k+1}}, & n \geqslant i+1 ; \quad \text { (2.7) } \\
\tilde{d}_{n}=\sum_{1 \leqslant k \leqslant n} \frac{\widetilde{F}_{n}^{(k)}}{q_{k, k+1}}, \quad(15.2) & \tilde{m}_{n}=\sum_{k=0}^{n} \frac{\widetilde{F}_{n}^{(k)}}{q_{k, k+1}}, \quad n \geqslant 0 .
\end{array}
$$

Relation of the three sequences:

$$
\tilde{m}_{n}=\frac{1}{q_{01}} \widetilde{F}_{n}^{(0)}+\tilde{d}_{n}, \quad n \geqslant 0 .
$$

